\title{
The canonical subgroup: a "subgroup-free" approach
}

\author{
Eyal Z. Goren and Payman L. Kassaei
}

\begin{abstract}
Beyond the crucial role they play in the foundations of the theory of overconvergent modular forms, canonical subgroups have found new applications to analytic continuation of overconvergent modular forms. For such applications, it is essential to understand various "numerical" aspects of the canonical subgroup, and in particular, the precise extent of its overconvergence. In this paper, we develop a theory of canonical subgroups for a general class of curves (including the unitary and quaternionic Shimura curves), using formal and rigid geometry. In our approach, we use the common geometric features of these curves rather than their (possible) specific moduli-theoretic description; it allows us to reproduce, for the classical cases, the optimal radii of definition for the canonical subgroup, usually derived by employing the theory of formal groups.
\end{abstract}

Mathematics Subject Classification (2000). Primary 11F85, 11F33; Secondary 11G18, 14G35, $14 \mathrm{G} 22$.

Keywords. Canonical subgroup, overconvergent modular form, rigid geometry.

\section{Introduction}

Canonical subgroups are essential to the theory of overconvergent modular forms. An elliptic curve $E$ with an ordinary reduction modulo a prime $p$ has a distinguished subgroup of rank $p$, which is the kernel of multiplication by $p$ on its formal group. This subgroup is a canonical lift of the kernel of $\operatorname{Fr}_{p}$ on $E$ modulo $p$. The overconvergence of the canonical subgroup, i.e. the fact that it can also be defined for elliptic curves with a "not too supersingular" reduction modulo $p$, allows one to define and study the $\mathbf{U}_{p}$ operator for overconvergent modular forms (See [Kat, §3.11].). Recently, in [Buz2], [Kas3], this theory has found new applications to the problem of analytic continuation of overconvergent modular forms. In these applications it is essential to understand the precise extent of overconvergence of the canonical subgroup, and to determine the "measure of supersingularity" of a quotient of an elliptic curve by a subgroup of order $p$ (including the canonical subgroup). These results appear in 
[Kat, Thms. 3.1, 3.10.7], where they are attributed to Lubin. A slightly more general version can be found in [Buz2].

Classically, the canonical subgroup of an elliptic curve (when it exists) is constructed by a close study of the power series of multiplication by $p$ in its formal group. In [Kas1], [Kas2] this approach was used to develop a similar theory over certain PEL Shimura curves. Generalizing this approach to higher dimensions seems to pose a serious challenge, because it uses the one-dimensionality of the formal group in an essential way, including the existence of Newton polygon for power series in one variable.

The problem of constructing a canonical subgroup for each elliptic curve belonging to a certain region of a modular curve $X(\Gamma)$ can be rephrased as finding a partial section to the forgetful morphism of rigid analytic curves $\pi: X\left(\Gamma_{0}(p) \cap \Gamma\right) \rightarrow X(\Gamma)$ whose moduli-theoretic description is $(E, \gamma, H) \mapsto(E, \gamma)$ where $(E, \gamma)$ is an elliptic curve with level $\Gamma$-structure and $H \subset E[p]$ is a finite flat subgroup of order $p$. Our approach ignores this moduli-theoretic description and just takes into account the geometry of the morphism $\pi$. This is what we call the "subgroup-free" approach. It has been known for a while that one can prove, using a general principle of rigid geometry due to Berthelot [Ber], that such a section defined over the ordinary locus overconverges (to an a priori non-tractable extent) beyond the ordinary locus. This approach, which is expected to work in other situations, was used in [KL] to prove the overconvergence of canonical subgroups in the case of Hilbert modular varieties. However, other aspects of the theory, which were discussed in the opening paragraph, remain unsettled even in the case of Hilbert modular varieties. These aspects are also not fully covered by other recent approaches [AM], [AG], [Con], [Nev].

The purpose of this article is to derive all aspects of the theory of canonical subgroups via the"subgroup-free" approach. Our thesis is that the rigid geometric (or formal schematic) picture that arises in the familiar setting of the relevant Shimura varieties suffices by itself to guarantee the existence of the canonical subgroup and many of its properties. In this manuscript we demonstrate that for Shimura varieties of dimension one, even if they do not possess a natural modular interpretation. In fact, this lack of a moduli interpretation can be taken as a further motivation for our approach. Notice that our approach is such that inspires generalization to higher dimensional settings. More specifically, one constructs a section over the ordinary locus by lifting a section from characteristic $p$. One separately studies sections over the non-ordinary locus by using the theory of local models for the special fibre of the Shimura variety in question, and finally these two sections are glued together by using the above-mentioned principle of rigid geometry along with a certain uniqueness result. The authors hope to pursue this subject in a future publication.

Let $p$ be a prime. Let $\mathcal{O}=\mathcal{O}_{K}$ be the ring of integers of a finite extension $K$ of $\mathbb{Q}_{p}, \varpi$ a uniformizer of $\mathcal{O}, \kappa=\mathcal{O} /(\varpi)$ the residue field, and val $=\mathrm{val}_{K}$ be the valuation normalized so that $\operatorname{val}(\varpi)=1$. By a "curve" $X$ over $\mathcal{O}$ we mean a 
flat finite-type morphism $f: X \rightarrow \mathcal{O}$ of relative dimension 1 of a reduced separated scheme $X$, such that the geometric fibres of $f$ are connected; $f$ need not be proper.

Let $X, Y$ be curves over $\mathcal{O}$. We assume that $X, Y$ are regular schemes, $X \rightarrow$ $\operatorname{Spec}(\mathcal{O})$ is smooth and $\pi: Y \rightarrow X$ is a finite flat morphism of degree $e+1$. Moreover, we assume that (i) there exists a section $s: X \otimes \kappa \rightarrow Y \otimes \kappa$ to $\pi \otimes \kappa$, that (ii) the special fibre $Y \otimes \kappa$ is a reduced normal crossing divisor with two components, and (iii) the set theoretic preimage $(\pi \otimes \kappa)^{-1}(\pi \otimes \kappa)(Q)$ is equal to $Q$ for any singular point $Q \in Y \otimes \kappa$. To remove any doubt, we assume that $Y \otimes \kappa$ is singular and by a normal crossing divisor we mean that each intersection is defined over $\kappa$ and its completed local ring is isomorphic to $\kappa \llbracket s, t \rrbracket /(s t)$. We define $(Y \otimes \kappa)^{\infty}=s(X \otimes \kappa) \backslash(Y \otimes \kappa)^{\text {sing }}$, and $(Y \otimes \kappa)^{0}=(Y \otimes \kappa) \backslash s(X \otimes \kappa)$.

From the point of view of a general theory this is a very specific situation, nonetheless it (and its appropriate generalization) is the one that occurs for Shimura curves (respectively, higher-dimensional PEL Shimura varieties); see §5. In fact, condition (iii) is only put to have "cleaner statements"; it holds in the case of Shimura curves. Under these conditions, we prove in $\S 3$ the following result.

Let $\mathfrak{X}, \mathfrak{Y}$ be the formal schemes obtained, respectively, by completing $X, Y$ along their special fibres. The induced morphism $\mathfrak{Y} \rightarrow \mathfrak{X}$ is still denoted by $\pi$. Let $\pi_{\text {rig }}: \mathfrak{Y}_{\text {rig }} \rightarrow \mathfrak{X}_{\text {rig }}$ be the induced morphism of rigid $K$-spaces à la Raynaud; c.f. $\$ 2.1$. In $\S 2.3$ we define a "measure of singularity" $\nu_{\mathfrak{X}}(P) \in \mathbb{Q}^{\geq 0}$ (respectively, $\nu_{\mathfrak{Y}}(Q) \in \mathbb{Q}^{\geq 0}$ ) of a point $P$ of $\mathfrak{X}_{\text {rig }}$ (respectively, $\mathfrak{Y}_{\text {rig }}$ ); the definition is modelled over the notion of measure of supersingularity for modular curves. For every interval $I \subset \mathbb{R}$ we have an admissible open set $\mathfrak{Y}_{\text {rig }} I$, whose closed point are $\left\{Q \in \mathfrak{Y}_{\text {rig }}: \nu_{\mathfrak{Y}}(Q) \in I\right\}$. The set $\mathfrak{X}_{\text {rig }} I$ is defined similarly. The following theorem is proven in $\S 3$.

Theorem A. Assume $e>1$. The morphism $\pi_{\text {rig }}: \mathfrak{Y}_{\text {rig }} \rightarrow \mathfrak{X}_{\text {rig }}$ admits a section

$$
\mathfrak{s}_{\text {rig }}: \mathfrak{X}_{\text {rig }}[0, e /(e+1)) \rightarrow \mathfrak{Y}_{\text {rig }} .
$$

This section is maximal, namely, it can not be extended to any connected admissible open properly containing $\mathfrak{X}_{\text {rig }}[0, e /(e+1))$.

The reader acquainted with the theory of canonical subgroups will recognize that this theorem implies the classical existence theorem for canonical subgroups over modular curves, including the further statement (that to the best of our knowledge is not recorded in the literature) that the region over which one defines the canonical subgroup is the maximal possible, even from the point of view of maps of rigid spaces. The following theorem, proven in $\S 4$, will also be familiar to that reader as giving the behavior of the measure of supersingularity upon passing to a quotient by a subgroup of order $p$. We introduce the following terminology: Let $Q \in \mathfrak{Y}_{\text {rig. }}$. We say that $Q$ is (i) canonical if $\nu_{\mathfrak{Y}}(Q)<e /(e+1)$; (ii) anti-canonical if $\nu_{\mathfrak{Y}}(Q)>e /(e+1)$; and (iii) too singular if $\nu_{\mathfrak{Y}}(Q)=e /(e+1)$. 
Theorem B. Let $w$ be an automorphism of $\mathfrak{Y}$ that permutes the two components of $\mathfrak{Y}$. We denote by $w$ also the induced automorphism of $\mathfrak{Y}$ rig and its effect of points by $Q \mapsto Q^{w}$.

(1) $\nu_{\mathfrak{X}}\left(\pi_{\mathrm{rig}} Q\right)=0 \Leftrightarrow v_{\mathfrak{X}}\left(\pi_{\mathrm{rig}} Q^{w}\right)=0$. In this case $Q$ is canonical if and only if $Q^{w}$ is anti-canonical.

(2) If $\nu_{\mathfrak{X}}\left(\pi_{\text {rig }} Q\right)<(e+1)^{-1}$ and $Q$ canonical, then $\nu_{\mathfrak{X}}\left(\pi_{\text {rig }} Q^{w}\right)=e \cdot v_{\mathfrak{X}}\left(\pi_{\text {rig }} Q\right)$ and $Q^{w}$ is anti-canonical.

(3) If $v_{\mathfrak{X}}\left(\pi_{\mathrm{rig}} Q\right)=(e+1)^{-1}$ and $Q$ is canonical then $Q^{w}$ is too singular.

(4) If $(e+1)^{-1}<v_{\mathfrak{X}}\left(\pi_{\text {rig }} Q\right)<e(e+1)^{-1}$ and $Q$ is canonical, then $v_{\mathfrak{X}}\left(\pi_{\text {rig }} Q^{w}\right)=$ $1-v_{\mathfrak{X}}\left(\pi_{\mathrm{rig}} Q\right)$ and $Q^{w}$ is canonical.

(5) If $v_{\mathfrak{X}}\left(\pi_{\mathrm{rig}} Q\right)<e(e+1)^{-1}$ and $Q$ is anti-canonical, then $v_{\mathfrak{X}}\left(\pi_{\mathrm{rig}} Q^{w}\right)=$ $e^{-1} v_{\mathfrak{X}}\left(\pi_{\text {rig }} Q\right)$, and $Q^{w}$ is canonical.

(6) If $Q$ is too singular then $v_{\mathfrak{X}}\left(\pi_{\mathrm{rig}} Q^{w}\right)=(e+1)^{-1}$ and $Q^{w}$ is canonical.

Acknowledgments. The authors benefited from an example of R. Coleman (private communication) that inspired the proof of Proposition 3.8. We also wish to thank the referees for a very careful reading of the manuscript and useful suggestions.

The first-named author was partially supported by an NSERC grant no. 227040 . The second-named author would like to thank CICMA and the department of mathematics at McGill university for their support and hospitality.

\section{Background material}

2.1. Rigid analytic varieties and formal schemes. We recall here the connection between rigid analytic varieties and formal schemes as developed by Raynaud and Berthelot. Our exposition follows [BLI], [BLII], [Ber], [deJ2].

Let $R$ be a valuation ring of Krull dimension 1, complete and separated with respect to the $\mathfrak{I}$-adic topology, where $\mathfrak{I}=(\varpi)$ is contained in the maximal ideal of $R$. Let $K$ be the field of fractions of $R$. For free variables $\xi=\left(\xi_{1}, \ldots, \xi_{n}\right)$ we let $R\langle\xi\rangle=\left\{\sum_{\nu} c_{\nu} \xi^{\nu} \in R \llbracket \xi \rrbracket: \lim c_{\nu}=0\right\}$ be the strictly convergent powerseries, i.e. precisely those that converge on the polydisc $\left\{\left(a_{1}, \ldots, a_{n}\right):\left|a_{i}\right| \leq 1\right.$ for all $\left.i\right\}$.

Recall that for a general commutative ring $B$ and an ideal $J$ of $B$ one defines the $J$-torsion of $B$ as the ideal $\left\{b \in B: J^{n} b=0\right.$ for some $\left.n \in \mathbb{N}\right\}$. If $J=\left(g_{1}, \ldots, g_{r}\right)$, the $J$-torsion is the kernel of the canonical homomorphism $R \rightarrow \prod_{i=1}^{r} R\left[g_{i}^{-1}\right]$. If this ideal is $\{0\}$ we say that $B$ has no $J$-torsion.

An admissible $R$-algebra is an $R$-algebra with no $\mathfrak{I}$-torsion (equivalently, flat over $R$ ) that is isomorphic to $R\langle\xi\rangle / \mathfrak{a}$, where $\xi=\left(\xi_{1}, \ldots, \xi_{n}\right)$ for some integer $n$; it implies that $\mathfrak{a}$ is a finitely generated ideal. For us, the admissible $R$-algebras are the 
building blocks of two different categories - a category of rigid spaces and a category of formal schemes.

An affine formal $R$-scheme $\mathfrak{X}$ is called admissible if it is of the type $\mathfrak{X}=\operatorname{Spf}(A)$, where $A$ is an admissible $R$-algebra. We may then write $\mathfrak{X}=\lim _{\lambda \rightarrow \infty} \mathfrak{X}_{\lambda}$, where $\mathfrak{X}_{\lambda}:=\mathfrak{X} \otimes\left(R /\left(\varpi^{\lambda}\right)\right), \lambda \in \mathbb{N}$, can be identified with the scheme $\operatorname{Spec}\left(A \otimes R /\left(\varpi^{\lambda}\right)\right)$. Being admissible is a local property and so one gets a natural definition of an admissible formal R-scheme.

The notion of admissible blow-up is needed to define an equivalence of categories between a category of formal schemes and a category of rigid spaces. The definition of admissible formal blow-up is designed to be local on the base. We review, thus, only the affine case. Let $\mathfrak{X}=\operatorname{Spf}(A)$ be an affine admissible $R$-formal scheme, $A=R\langle\xi\rangle / \mathfrak{a}$. Let $\mathscr{A}$ be an open ideal, i.e., containing $\left(\varpi^{\lambda}\right)$ for some $\lambda>0$. The admissible formal blow-up of $\mathfrak{X}$ at $\mathscr{A}$ is $\mathfrak{X}^{\prime}=\lim _{\lambda \rightarrow \infty} \operatorname{Proj} \bigoplus_{n=0}^{\infty}\left(\mathscr{A}^{n} \otimes \mathcal{O}_{\mathfrak{X}} /\left(\varpi^{\lambda}\right)\right)$ with the canonical map $\varphi: \mathfrak{X}^{\prime} \rightarrow \mathfrak{X}$. Then $\mathfrak{X}^{\prime}$ is an admissible formal $R$-scheme over which $\mathscr{A} \mathcal{O}_{\mathfrak{X}^{\prime}}$ is invertible.

Let $\mathscr{A}=\left(f_{0}, \ldots, f_{m}\right)$ and let $\widetilde{\varphi}: \widetilde{\mathfrak{X}}^{\prime} \rightarrow \widetilde{\mathfrak{X}}=\operatorname{Spec}(A)$ be the usual scheme theory blow-up of $A$ at the ideal $\mathscr{A}$. Then, upon taking $(\varpi)$-completion of $\widetilde{\varphi}: \widetilde{\mathfrak{X}}^{\prime} \rightarrow \widetilde{\mathfrak{X}}$ we get $\varphi: \mathfrak{X}^{\prime} \rightarrow \mathfrak{X}$. On the other hand, $\widetilde{\varphi}: \widetilde{\mathfrak{X}^{\prime}} \rightarrow \widetilde{\mathfrak{X}}$ admits a local description. The scheme $\tilde{\mathfrak{X}}^{\prime}$ has an affine cover by $\left\{\operatorname{Spec}\left(A_{i}^{\prime}\right): i=0,1, \ldots, m\right\}$, where $A_{i}^{\prime}=A_{i}^{\prime \prime} /\left(f_{i}-\right.$ torsion $)$ and $A_{i}^{\prime \prime}=A\left[\frac{f_{0}}{f_{i}}, \ldots, \frac{f_{m}}{f_{i}}\right]=A\left[\frac{\zeta_{0}}{\zeta_{i}}, \ldots, \frac{\zeta_{m}}{\zeta_{i}}\right] /\left(f_{i} \frac{\zeta_{j}}{\zeta_{i}}-f_{j}\right)$. To clarify, in the definition of $A_{i}^{\prime}$ (and similarly below), the notation $\left(f_{i}-\right.$ torsion)

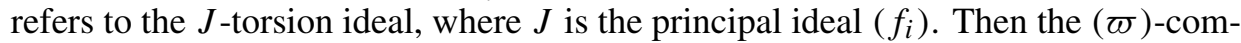
pletions of $A_{i}^{\prime}, A_{i}^{\prime \prime}$ are given by $\hat{A}_{i}^{\prime}=\hat{A}_{i}^{\prime \prime} /\left(f_{i}-\right.$ torsion $)$ and $\hat{A}_{i}^{\prime \prime}=A\left\langle\frac{f_{0}}{f_{i}}, \ldots, \frac{f_{m}}{f_{i}}\right\rangle=$ $A\left(\frac{\zeta_{0}}{\zeta_{i}}, \ldots, \frac{\zeta_{m}}{\zeta_{i}}\right) /\left(f_{i} \frac{\zeta_{j}}{\zeta_{i}}-f_{j}\right)$; they give rise to an affine covering $\left\{\operatorname{Spf}\left(\hat{A}_{i}^{\prime}\right): i=\right.$ $0,1, \ldots, m\}$ of $\mathfrak{X}^{\prime}$.

For an admissible $R$-algebra $A=R\langle\xi\rangle / \mathfrak{a}$, let $A_{\text {rig }}:=A \otimes_{R} K=K\langle\xi\rangle / \mathfrak{a} K\langle\xi\rangle$; this is an affinoid $K$-algebra. This construction extends to provide a functor

rig: $\{$ admissible formal $R$-schemes $\} \rightarrow$ rigid $K$-spaces $\}, \quad \mathfrak{X} \mapsto \mathfrak{X}_{\text {rig. }}$.

One calls $\mathfrak{X}_{\text {rig }}$ the generic fibre of the formal $R$-scheme $\mathfrak{X}$.

Theorem 2.1 (Raynaud). The functor rig is an equivalence of categories between (i) the category of quasi-compact admissible formal R-schemes, localized by admissible formal blow-ups, and (ii) the category of quasi-compact and quasi-separated rigid $K$-spaces.

It is easy to see from the construction that a flat morphism of formal schemes induces a flat morphism of rigid spaces. The converse is also true [BLII, Thm. 5.2]: every flat morphism of rigid $K$-spaces comes from a flat morphism of suitable formal 
schemes yielding the given rigid spaces. A flat morphism in the category of rigid spaces has image which is a finite union of affinoids, in particular, it is open [BLII, Cor. 5.11].

We will need to use the specialization map. In the affine case, the points of $\mathfrak{X}_{\text {rig }}$ are the maximal ideals of the algebra $A \otimes_{R} K$; these are in bijection with quotients of $A$ that are integral, finite and flat over $R$. If $T$ is such a quotient (it is the valuation ring of a finite extension of $K$ ), corresponding to a point $t \in \mathfrak{X}_{\text {rig }}$, we get a closed immersion of formal $R$-schemes $\operatorname{Spf}(T) \rightarrow \operatorname{Spf}(A)$, whose image is supported on a closed point of $\mathfrak{X}$ that we denote by $\operatorname{sp}(t)$. The definition can be extended to any formal $R$-scheme. We get a morphism of ringed spaces sp: $\mathfrak{X}_{\text {rig }} \rightarrow \mathfrak{X}$ [SGA4, IV 4.9]. For every affine open $U=\operatorname{Spf}(B) \subset \mathfrak{X}$, we have $\operatorname{sp}^{-1}(U)=U_{\text {rig }}$.

Assume that $R$ is a discrete valuation ring with residue field $\kappa$. In [Ber] Berthelot generalizes the above construction to associate a generic fibre to any locally noetherian formal scheme $\mathfrak{X}$ flat over $R$ that satisfies a condition weaker than admissibility: that the special fibre of $\mathfrak{X}$, denoted by $\mathfrak{X}_{0}$ and defined by the ideal of definition $\mathscr{I}$, is a scheme locally of finite type over $\kappa$. This condition is independent of the choice of $\mathscr{I}$ and coincides with admissibility if $\varpi \mathcal{O}_{\mathfrak{X}}$ is an ideal of definition for $\mathfrak{X}$. We will describe the construction in the affine case. Let $\mathfrak{X}=\operatorname{Spf}(A)$ and $I=H^{0}(\mathfrak{X}, \mathscr{I})$ with generators $g_{1}, \ldots, g_{r}$. For $n \geq 1$ define

$$
A_{n}=A\left\langle T_{1}, \ldots, T_{r}\right\rangle /\left(g_{1}^{n}-\varpi T_{1}, \ldots, g_{r}^{n}-\varpi T_{r}\right) .
$$

The condition on $\mathfrak{X}$ implies that $A_{n} / \varpi A_{n}$ is finitely generated over $\kappa$, and hence $\mathfrak{X}^{n}=$ $\operatorname{Spf}\left(A_{n}\right)$ is an admissible formal scheme over $R$. Applying Raynaud's construction we obtain a rigid analytic space $\mathfrak{X}_{\text {rig. }}^{n}$. For $m>n$ we have a homomorphism $A_{m} \rightarrow A_{n}$, defined by sending $T_{i}$ to $g_{i}^{m-n} T_{i}$, inducing a morphism of rigid spaces $\mathfrak{X}_{\text {rig }}^{n} \rightarrow \mathfrak{X}_{\text {rig }}^{m}$. It is easy to see that this morphism is an open immersion and identifies $\mathfrak{X}_{\text {rig }}^{n}$ with the subdomain of $\mathfrak{X}_{\text {rig }}^{m}$ over which $\left|g_{i}(x)\right| \leq|\varpi|^{1 / n}$. The generic fibre of $\mathfrak{X}$, denoted as before by $\mathfrak{X}_{\text {rig }}$, is defined to be the union of $\mathfrak{X}_{\text {rig }}^{n}$ via the above inclusions. The rigid spaces $\mathfrak{X}_{\text {rig }}^{n}$ form an admissible cover of $\mathfrak{X}_{\text {rig. }}$. The construction yields a functor rig whose target is the category of quasi-separated rigid $K$-spaces.

As an illustration, take $\mathfrak{X}$ to be $\operatorname{Spf}\left(R \llbracket \xi_{1}, \ldots, \xi_{r} \rrbracket\right)$ with the ideal of definition $I=\left(\varpi, \xi_{1}, \ldots, \xi_{r}\right)$. Then $\mathfrak{X}_{\text {rig }}$ is simply the open unit polydisc of dimension $r$, which is not quasi-compact, and $\mathfrak{X}_{\text {rig }}^{n} \subset \mathfrak{X}_{\text {rig }}$ is the affinoid subdomain over which $\left|\xi_{i}\right| \leq|\varpi|^{1 / n}$, which is isomorphic to a closed unit polydisc, and hence is quasicompact. Similarly, for $\mathfrak{X}=\operatorname{Spf}\left(R \llbracket x_{1}, x_{2} \rrbracket /\left(x_{1} x_{2}-a\right)\right)$, where $a \in R$, one sees that $\mathfrak{X}_{\text {rig }}$ is the open annulus over $K$ with radii $(|a|, 1)$.

As in the admissible case, one can define a specialization map sp: $\mathfrak{X}_{\text {rig }} \rightarrow \mathfrak{X}$ by taking the direct limit of the maps $\mathfrak{X}_{\text {rig }}^{n} \stackrel{\text { sp }}{\longrightarrow} \mathfrak{X}^{n} \rightarrow \mathfrak{X}$. The following is Proposition 0.2.7. of [Ber]. 
Proposition 2.2. Let notation be as above. Let $\mathfrak{Z} \subseteq \mathfrak{X}_{0}$ be a closed subscheme. Let $\mathfrak{X}^{\wedge \mathfrak{Z}}$ denote the formal completion of $\mathfrak{X}$ along $\mathfrak{Z}$. Then $\mathrm{sp}^{-1}(\mathfrak{Z})$ is an admissible open subset of $\mathfrak{X}_{\text {rig }}$ and the canonical morphism $\mathfrak{X}_{\text {rig }}^{\wedge \mathfrak{Z}} \rightarrow \mathfrak{X}_{\text {rig }}$ induces a functorial isomorphism $\mathfrak{X}_{\text {rig }}^{\wedge \mathfrak{Z}} \cong \mathrm{sp}^{-1}(\mathfrak{Z})$.

2.2. Algebraic geometric input. As in the Introduction, let $\mathcal{O}$ be the ring of integers of a finite extension $K$ of $\mathbb{Q}_{p}$, $\varpi$ a uniformizer of $\mathcal{O}$ and $\kappa=\mathcal{O} /(\varpi)$ the residue field. Let $X, Y$ be relative curves over $\mathcal{O}$. We assume that $X, Y$ are regular schemes, $X \rightarrow \operatorname{Spec}(\mathcal{O})$ is smooth and $\pi: Y \rightarrow X$ is a finite flat morphism of degree $e+1$. Moreover, we assume that (i) there exists a section $s: X \otimes \kappa \rightarrow Y \otimes \kappa$ to $\pi \otimes \kappa$, that (ii) the special fibre $Y \otimes \kappa$ is a reduced normal crossing divisor with two components, and that (iii) the set theoretic preimage $(\pi \otimes \kappa)^{-1}(\pi \otimes \kappa)(Q)$ is equal to $Q$ for any singular point $Q \in Y \otimes \kappa$.

The following lemma must be known to the experts; for lack of a reference we provide a proof.

Lemma 2.3. Let $(A, \mathfrak{m})$ be a regular two-dimensional complete local ring containing $\mathcal{O}$, such that $\mathcal{O}$ is integrally closed in $A, \mathfrak{m} \cap \mathcal{O}=(\varpi)$, and $\kappa \subseteq A / \mathfrak{m}$ is an algebraic extension.

(1) If $A \otimes \kappa$ is regular then $A \cong \mathcal{O} \llbracket x \rrbracket$.

(2) If $A \otimes \kappa \cong \kappa \llbracket s, t \rrbracket /(s t)$ then $A \cong \mathcal{O} \llbracket x, y \rrbracket /(x y-\varpi)$.

Proof. First note that $A / \mathfrak{m} \supseteq \kappa$ and so the local homomorphism $W(A / \mathfrak{m}) \rightarrow A$ has image containing $W(\kappa)$ viewed as a subring of $\mathcal{O}$. Since $A / \mathfrak{m}$ is an algebraic extension of $\kappa, W(A / \mathfrak{m})$ is integral over $W(\kappa)$. Since $\mathcal{O}$ is integrally closed in $A$ it follows that $W(A / \mathfrak{m})$ is contained in $\mathcal{O}$. In particular, $A / \mathfrak{m}=\kappa$.

If $A \otimes \kappa$ is regular it follows by Cohen's Theorem that $A \otimes \kappa \cong \kappa \llbracket x \rrbracket$. This gives a morphism $\mathcal{O} \llbracket x \rrbracket \rightarrow A$ which is surjective by Nakayama's lemma; since both rings are domains of the same dimension, we conclude that $\mathcal{O} \llbracket x \rrbracket \rightarrow A$ is an isomorphism (the kernel is a prime ideal of height 0 ).

Assume then that $A \otimes \kappa \cong \kappa \llbracket s, t \rrbracket /(s t)$. Let $x^{\prime}, y^{\prime} \in A$ be elements reducing to $s, t$, respectively. The homomorphism $\mathcal{O} \llbracket x, y \rrbracket \rightarrow A$, taking $x, y$ to $x^{\prime}, y^{\prime}$ respectively, is surjective by Nakayama's lemma. Let $\mathfrak{p}$ be the kernel; it is a prime ideal of height 1 . In fact $\mathfrak{p}$ is a principal ideal, because $\mathcal{O} \llbracket x, y \rrbracket$ is a factorial ring and by a theorem of Krull every prime ideal of height 1 is principal. We may therefore write $\mathfrak{p}=(h(x, y))$, where $h(x, y)=x y v-\varpi z$ for some $v, z \in \mathcal{O} \llbracket x, y \rrbracket$. It follows that $A \otimes \kappa \cong \kappa \llbracket x, y \rrbracket /(x y \bar{v})$, where $\bar{v}$ is the reduction of $v$ modulo $\varpi$. Since $\kappa \llbracket x, y \rrbracket /(x y \bar{v}) \cong \kappa \llbracket s, t \rrbracket /(s t)$ by the map taking $x \mapsto s$ and $y \mapsto t$, it follows that $\bar{v}$ is a unit. This implies that $v$ itself is a unit and so $A \cong \mathcal{O} \llbracket x, y \rrbracket /(x y-\varpi z)$.

We next claim that the ring $A$ is regular if and only if $z$ is a unit. Indeed, if $z$ is a unit then $A \cong \mathcal{O} \llbracket x, y z^{-1} \rrbracket /\left(x \cdot y z^{-1}-\varpi\right)$, which is easily checked to be 
regular. Assume now that $A$ is regular. Then $(\varpi, x, y) / I$ is a 2 -dimensional $\kappa=$ $A / \mathfrak{m}$ vector space, where $I=(\varpi, x, y)^{2}+(x y-\varpi z)$ and $\mathfrak{m}$, the maximal ideal of $A$, is the image of $(\varpi, x, y)$. So, for some $c_{1}, c_{2}, c_{3} \in A$, not all in $\mathfrak{m}$, we have $c_{1} \varpi+c_{2} x+c_{3} y \in I$. Such a relation gives modulo $\varpi$ the relation $c_{2} x+c_{3} y \in$ $(x, y)^{2}$. Since the cotangent space at the singular point is two dimensional with basis $\{x, y\}$, it follows that modulo $\varpi$ we have $c_{2}, c_{3} \in(x, y)$. Thus, we must have $c_{2}, c_{3} \in \mathfrak{m}$. Therefore, $A$ is regular implies that $\varpi \in I$. Thus, $\varpi\left(\bmod \mathfrak{m}^{2}\right) \in$ $I / \mathfrak{m}^{2}=(\varpi z) / \mathfrak{m}^{2}$. It follows that $z$ is a unit modulo $\mathfrak{m}^{2}$ and hence is a unit.

Lemma 2.4. Let $Q \in Y$ be a singular point and $P=\pi(Q)$. There is a choice of local coordinates at $Q$ and $P$ giving $\mathcal{O}_{Y}^{\wedge Q} \cong \mathcal{O} \llbracket x, y \rrbracket /(x y-\varpi)$ and $\mathcal{O}_{X}^{\wedge} \cong \mathcal{O} \llbracket t \rrbracket$, respectively, such that on the level of completed local rings at $Q$ and $P$ the morphism $\pi$ is given by

$$
t \mapsto x+(y u)^{e}+f(y)+\varpi g,
$$

where $f(y) \equiv 0\left(\bmod \left(y^{e+1}\right)\right)$ and $u$ is a unit congruent to 1 modulo $\varpi$.

Proof. It follows from Lemma 2.3 that the map $Y \rightarrow X$ can be written at a singular point $Q \in Y$ in the form of an $\mathcal{O}$-algebra local homomorphism $\pi^{*}: \mathcal{O} \llbracket t \rrbracket \rightarrow$ $\mathcal{O} \llbracket x, y \rrbracket /(x y-\varpi)$. Now, upon reduction modulo $\varpi$, we get a homomorphism of $\kappa$ algebras $\pi^{*} \otimes \kappa: \kappa \llbracket t \rrbracket \rightarrow \kappa \llbracket x, y \rrbracket /(x y)$. By our assumptions on $\pi \otimes \kappa$, the compositions $\kappa \llbracket t \rrbracket \rightarrow \kappa \llbracket x, y \rrbracket /(x y) \underset{y \mapsto 0}{\longrightarrow} \kappa \llbracket x \rrbracket$ and $\kappa \llbracket t \rrbracket \rightarrow \kappa \llbracket x, y \rrbracket /(x y) \underset{x \mapsto 0}{\longrightarrow} \kappa \llbracket y \rrbracket$ are given, w.l.o.g., by $t \mapsto x$ and $t \mapsto y^{e}+f_{1}(y)$, where $f_{1}(y) \equiv 0 \bmod \left(y^{e+1}\right)$ (the existence of the section implies that every ramification index is equal to $e$ ). Thus, the map $\pi^{*} \otimes \kappa$ is determined by the image of $t$ which has the form $x+y^{e}+f_{1}(y)+$ $x y f_{2}(x, y)$.

Our goal now is to change coordinates on $A:=\mathcal{O} \llbracket x, y \rrbracket /(x y-\varpi)$ so as to simplify this map and still have the same presentation, namely, find $\hat{x}, \hat{y} \in A$ such that $\mathcal{O} \llbracket x, y \rrbracket /(x y-\varpi)=\mathcal{O} \llbracket \hat{x}, \hat{y} \rrbracket /(\hat{x} \hat{y}-\varpi)$. First note that since $A$ is $\varpi-$ adically complete the map of units $A^{\times} \rightarrow(A \otimes \kappa)^{\times}$is surjective. Let $u^{\prime}=$ $\left(1+y f_{2}(x, y)\right) \in(A \otimes \kappa)^{\times}$and $\hat{u}$ any lift of it to $A^{\times}$. Let $\hat{x}=x \hat{u}, \hat{y}=y \hat{u}^{-1}$. Then we have $\mathcal{O} \llbracket \hat{x}, \hat{y} \rrbracket(\hat{x} \hat{y}-\varpi)=\mathcal{O} \llbracket x, y \rrbracket /(x y-\varpi)$ and the map $\mathcal{O} \llbracket t \rrbracket \rightarrow$ $\mathcal{O} \llbracket \hat{x}, \hat{y} \rrbracket /(\hat{x} \hat{y}-\varpi)$ has the form $t \mapsto \hat{x}+(\hat{y} \hat{u})^{e}+\hat{f}(\hat{y})+\varpi \hat{g}$, where $\hat{f}$ is a lift of $f_{1}$ satisfying $\hat{f}(y) \equiv 0\left(\bmod \left(y^{e+1}\right)\right)$.

2.3. A measure of singularity. Let $\pi: Y \rightarrow X$ be a morphism of curves as in $\S 2.2$. We denote by $\mathfrak{X}, \mathfrak{Y}$ the formal schemes obtained from $X, Y$ by completion along their special fibres. Let $\beta_{1}, \ldots, \beta_{h}$ be the singular points of $Y$. Let $\alpha_{i}=\pi\left(\beta_{i}\right)$ for $i=1, \ldots, h$. Recall that by assumption the $\alpha_{i}$ 's and $\beta_{i}$ 's are defined over $\kappa$. Let $D_{\alpha_{i}}$ (respectively $D_{\beta_{i}}$ ) denote the inverse image of $\alpha_{i}$ (respectively $\beta_{i}$ ) under the specialization map sp: $\mathfrak{X}_{\text {rig }} \rightarrow \mathfrak{X}$ (respectively sp: Y $\left.\mathfrak{Y}_{\text {rig }} \rightarrow \mathfrak{Y}\right)$. 
By Proposition 2.2 $D_{\alpha_{i}}$ is the rigid space associated to $\operatorname{Spf}\left(\mathcal{O}_{X}^{\wedge \alpha_{i}}\right) \cong \operatorname{Spf}(\mathcal{O} \llbracket t \rrbracket)$, using Lemma 2.3. Therefore $D_{\alpha_{i}}$ is an open disc of radius 1 with parameter $t$. This parameter is unique up to $t \mapsto t^{\prime}=t \epsilon+\varpi z$, where $\epsilon \in \mathcal{O}^{\times}$and $z \in \mathcal{O} \llbracket t \rrbracket$. For a general closed point $P \in D_{\alpha_{i}}$ the value $\operatorname{val}(t(P))$ depends on $t$, however, if $\operatorname{val}(t(P))<1$ then $\operatorname{val}(t(P))=\operatorname{val}\left(t^{\prime}(P)\right)$ for any $t^{\prime}$ as above. We abuse notation and define

$$
v_{\mathfrak{X}}(P)=\operatorname{val}(t(P)),
$$

bearing in mind that this is well defined only if $\operatorname{val}(t(P))<1$.

Similarly, $D_{\beta_{i}}$ is the rigid space of $\operatorname{Spf}\left(\mathcal{O}_{X}^{\wedge \beta_{i}}\right) \cong \operatorname{Spf}(\mathcal{O} \llbracket x, y \rrbracket /(x y-\varpi))$. Therefore, $D_{\beta_{i}}$ is an open annulus of radii $(|\varpi|, 1)$ with parameter $x$. For any closed point $Q$ in $D_{\beta_{i}}$, define

$$
\nu_{\mathfrak{Y}}(Q)=\operatorname{val}(x(Q)) .
$$

This definition is independent of the choice of the parameters if chosen as in Lemma 2.4. The reason is that any other such parameter $x^{\prime}$ is of the form $x^{\prime}=x \epsilon+\varpi z$, where $\epsilon \in \mathcal{O}^{\times}$and $z \in \mathcal{O} \llbracket x, y \rrbracket /(x y-\varpi)$, and $\operatorname{val}(x(Q))<1$.

Let $\mathscr{Z}$ denote the complement in $\mathfrak{X}_{\text {rig }}$ of $\operatorname{sp}^{-1}\left(\left\{\alpha_{1}, \ldots, \alpha_{h}\right\}\right)$. For a closed point $P$ in $\mathscr{Z}$ we define $v_{\mathfrak{X}}(P)=0$. By Proposition 3.1 below, the complement in $\mathfrak{Y}_{\text {rig }}$ of $\mathrm{sp}^{-1}\left(\left\{\beta_{1}, \ldots, \beta_{h}\right\}\right)$ has two connected components,

and

$$
\mathscr{Z}^{0}=\operatorname{sp}^{-1}\left((Y \otimes \kappa)^{0}-\left\{\beta_{1}, \ldots, \beta_{h}\right\}\right),
$$

$$
\mathscr{Z}^{\infty}=\mathrm{sp}^{-1}\left((Y \otimes \kappa)^{\infty}-\left\{\beta_{1}, \ldots, \beta_{h}\right\}\right) .
$$

For points in $\mathscr{Z}^{\infty}$ we define $\nu_{\mathfrak{Y}}$ to be 0 , and on $\mathscr{Z}^{0}$ we define $\nu_{\mathfrak{Y}}$ to be 1 . We refer to $v_{\mathfrak{X}}$ and $\nu_{\mathfrak{Y}}$ as measures of singularity. For an interval $I$ of real numbers, we define $\mathfrak{X}_{\text {rig }} I$ to be the set of points of $\mathfrak{X}_{\text {rig }}$ where $\nu_{\mathfrak{X}}$ belongs to $I$. For $U$ an admissible open subset of $\mathfrak{X}_{\text {rig }}$ we set $U I=U \cap \mathfrak{X}_{\text {rig }} I$. We use a similar notation for $\mathfrak{Y}_{\text {rig }}$. We call $\mathscr{Z}$ the ordinary locus of $\mathfrak{X}_{\text {rig }}$ and its complement $\operatorname{sp}^{-1}\left(\left\{\alpha_{1}, \ldots, \alpha_{h}\right\}\right)=$ $\mathfrak{X}_{\text {rig }}(0, \infty)$ the singular locus of $\mathfrak{X}_{\text {rig. }}$. We have $\pi_{\text {rig }}{ }^{-1}\left(\mathfrak{X}_{\text {rig }}(0, \infty)\right)=\mathfrak{Y}_{\text {rig }}(0,1)=$ $\mathrm{sp}^{-1}\left(\left\{\beta_{1}, \ldots, \beta_{h}\right\}\right)$ which we call the singular locus of $\mathfrak{Y}_{\text {rig }}$.

\section{Main theorem}

In this section we prove Theorem A of the Introduction, using the same notation. Our strategy is to construct sections separately on the ordinary locus and the singular locus and glue them by means of a general principle of rigid geometry. We start by constructing a section to $\pi_{\text {rig }}$ over the ordinary locus of $\mathfrak{X}_{\text {rig }}$. 
Proposition 3.1. The map $\pi_{\text {rig }}$ induces an isomorphism between $\mathscr{Z}^{\infty}$ and $\mathscr{Z}$. Therefore there is a unique section $\mathfrak{s}_{\text {rig }}^{\infty}: \mathscr{Z} \rightarrow \mathfrak{Y}_{\text {rig }}$ to $\pi_{\text {rig }}$ whose image is $\mathscr{Z} \infty$. Furthermore, both $\mathscr{Z}^{\infty}$ and $\mathscr{Z}^{0}$ are connected. If $e>1$, then any section to $\pi_{\mathrm{rig}}$ on $\mathscr{Z}$ coincides with $\mathfrak{s}_{\text {rig }}^{\infty}$

Proof. We show the existence of the section on the level of the formal schemes. The curves $(Y \otimes \kappa)^{\infty},(Y \otimes \kappa)^{0}$ are connected reduced affine curves. Let $U$ be the open subset of $\mathfrak{Y}$ equal to the underlying set of $(Y \otimes \kappa)^{\infty} \cup(Y \otimes \kappa)^{0}$. Then $U$ is affine in the formal schemes sense, namely, we have an open immersion $\operatorname{Spf}(B) \rightarrow \mathfrak{Y}$ whose set theoretic image is $U$. Under the specialization map sp: $\mathfrak{Y}_{\text {rig }} \rightarrow \mathfrak{Y}$ we have $\operatorname{sp}^{-1}(U)=\mathscr{Z}^{0} \cup \mathscr{Z}^{\infty}$ and, moreover, $\mathscr{Z}^{0} \cup \mathscr{Z}^{\infty}=U_{\text {rig }}$ (c.f. the discussion in $\S 2.1)$. We conclude the following: We have a morphism $\operatorname{Spf}(B) \rightarrow \operatorname{Spf}(A)$, induced by a homomorphism of $\varpi$-adically complete $\mathcal{O}$-algebras $A \rightarrow B$, that yields the morphism $\mathscr{Z}^{0} \cup \mathscr{Z}^{\infty} \rightarrow \mathscr{Z}$ and reduces to the morphism $Y \otimes \kappa \backslash\left\{\beta_{i}\right\}_{i=1}^{h} \rightarrow$ $X \otimes \kappa \backslash\left\{\alpha_{i}\right\}_{i=1}^{h}$. It transpires that $B \otimes \kappa=(A \otimes \kappa) \oplus B_{1}$. Using Hensel's lemma to lift idempotents, we conclude that we have $B=A^{+} \oplus B_{1}^{+}$, with $A^{+} \otimes \kappa=$ $A \otimes \kappa, B_{1}^{+} \otimes \kappa=B_{1}$. Using that $A \rightarrow A^{+}$is a finite flat homomorphism reducing to an isomorphism after $\otimes \kappa$, we conclude that $A=A^{+}$. This gives the existence of the section $\mathfrak{s}^{\infty}: \operatorname{Spf}(A) \rightarrow \operatorname{Spf}(B)$, the analytification of which is the desired section $\mathfrak{s}_{\text {rig }}^{\infty}: \mathscr{Z} \rightarrow \mathscr{Z}^{0} \cup \mathscr{Z}^{\infty}$ with image $\mathscr{Z}^{\infty}$. In particular, $\mathscr{Z}^{\infty}$, being isomorphic to $\mathscr{Z}$, which is a curve minus finitely many residue discs, is connected.

Furthermore, the morphism $\operatorname{Spf}\left(B_{1}^{+}\right) \rightarrow \operatorname{Spf}(A)$ is finite flat of degree $e$. To show $\mathscr{Z}^{0}$ is connected it is enough to show that $\operatorname{Spf}\left(B_{1}^{+}\right)$is flat over $\operatorname{Spf}(\mathcal{O})$, and has a reduced and connected special fibre (see Remark 3.2). But this is clear since $\operatorname{Spec}\left(B_{1}^{+} \otimes \kappa\right)=(Y \otimes \kappa)^{0}$.

For the final assertion, note that the image of any section to $\pi_{\text {rig }}$ on $\mathscr{Z}$ must be a connected component of $\pi_{\text {rig }}{ }^{-1}(\mathscr{Z})=\mathscr{Z}^{\infty} \cup \mathscr{Z}^{0}$, and hence it must be either $\mathscr{Z}^{\infty}$ or $\mathscr{Z}^{0}$. The latter cannot happen since $\pi_{\text {rig }}: \mathscr{Z}^{0} \rightarrow \mathscr{Z}$ is $e$-to- 1 and $e>1$.

Remark 3.2. Let $\mathfrak{B}=\operatorname{Spf}(B)$ be an admissible formal scheme, with associated rigid space $\mathfrak{B}_{\text {rig. }}$. It is possible that $\mathfrak{B}_{\text {rig }}$ is disconnected, yet the underlying topological space of $\mathfrak{B}$ is connected. An example is provided when $\mathcal{O}$ is a ramified extension of $\mathbb{Z}_{p}$ and we let $B=\mathcal{O}\langle x, y, T\rangle /(x y-p,(x+y) T-\varpi)$. The associated rigid space is a disjoint union of two annuli. The special fibre is three lines meeting at a single point. Note though that $B \otimes \kappa=k[x, y, T] /(x y,(x+y) T)$ in which $x T$ is nilpotent.

On the other hand, assume $\mathfrak{B}$ is an admissible formal scheme over $\mathcal{O}$ such that $\mathfrak{B}_{\text {rig }}$ is affinoid (in particular $\mathfrak{B}_{\text {rig }}=\operatorname{Spm}\left(B \otimes_{\mathcal{O}} K\right)$ where $B=H^{0}\left(\mathfrak{B}, \mathcal{O}_{\mathfrak{B}}\right)$ ). If $\mathfrak{B} \otimes \kappa$

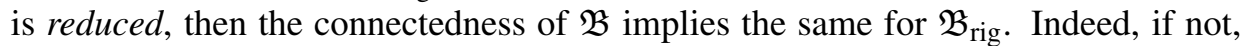
then there is a non-trivial idempotent element $e \in B \otimes_{\mathcal{O}} K$. We show that $e \in B$. Note that by flatness of $\mathfrak{B}$ over $\mathcal{O}$ we know that $B \hookrightarrow B \otimes K$. If $e \notin B$, we can 
write $e=f / \varpi^{n}$, where $n>0$ is minimal, and $f \in B$. Then we have $f^{2}=\varpi^{n} f$. Reducing modulo $\varpi$, we get $\bar{f} \neq 0$ and $\bar{f}^{2}=0$ which contradicts our assumption on $\mathfrak{B} \otimes \kappa$. Therefore $e \in B$. It then follows that the decomposition of the "generic fibre" $B \otimes K$, namely of the rigid space, induces a decomposition of the formal scheme $\operatorname{Spf}(B)$.

Let $\mathcal{X}$ be a $K$-rigid analytic space, and $U \subset \mathcal{X}$ be an admissible affinoid subdomain. An affinoid subdomain $U \subset \mathcal{V} \subset \mathcal{X}$ is called a strict neighborhood of $\mathcal{U}$ in $\mathcal{X}$ if the reduction of the inclusion $\iota: U \rightarrow \mathcal{V}$ factors through an affine scheme which is finite over $\operatorname{Spec}(\kappa)$. See [CGJ, §3] for more details. Any strict neighborhood of $\mathscr{Z}$ in $\mathfrak{X}_{\text {rig }}$ contains a domain of the form $\mathfrak{X}_{\text {rig }}[0, a]$ for some positive $a \in \mathbb{Q}$; c.f. [KL, Prop. 2.3.2]. The following is Lemma 6 of [CGJ]. See also [Ber].

Lemma 3.3. Let $f: \mathcal{Y} \rightarrow \mathcal{X}$ be a finite flat morphism of rigid analytic curves. Let $u$ be an affinoid subdomain of $\mathcal{X}$, and $s: \mathcal{U} \rightarrow \mathcal{Y}$ a section to $f$. Then $s$ can be extended to a strict neighborhood of $\mathcal{U}$ in $\mathcal{X}$.

Corollary 3.4. The section $\mathfrak{s}_{\text {rig }}^{\infty}$ extends to a section $\mathfrak{s}_{\text {rig }}^{\dagger}$ over $\mathfrak{X}_{\text {rig }}[0$, a] for some positive $a \in \mathbb{Q}$.

Next we discuss sections to $\pi_{\text {rig }}$ over the singular locus, i.e. where $\nu_{\mathfrak{X}}>0$.

Proposition 3.5. The map $\pi_{\text {rig }}: \mathfrak{Y}_{\text {rig }}(0,1) \rightarrow \mathfrak{X}_{\text {rig }}(0, \infty)$ admits a section $\mathfrak{t}$ on $\mathfrak{X}_{\text {rig }}(0, e /(e+1))$ whose image is $\mathfrak{Y}_{\text {rig }}(0, e /(e+1))$. Such a section is unique. If $e>1$, then we have the following stronger uniqueness result: any section to $\pi_{\mathrm{rig}}$ on a connected admissible open subset of $\mathfrak{X}_{\mathrm{rig}}(0, e /(e+1))$ which contains some circle $D_{\alpha_{i}}[a, a]$ is obtained by the restriction of $\mathrm{t}$.

Proof. We have $\mathfrak{Y}_{\text {rig }}(0,1)=\bigsqcup_{i} D_{\beta_{i}}$ and $\mathfrak{X}_{\text {rig }}(0, \infty)=\bigsqcup_{i} D_{\alpha_{i}}$. Since by our assumptions $\pi^{-1}\left(\alpha_{i}\right)=\left\{\beta_{i}\right\}$ as sets, we have $\pi_{\text {rig }}{ }^{-1}\left(D_{\alpha_{i}}\right)=D_{\beta_{i}}$, and hence, for the first assertion, it suffices to show that for each $i$ the map $\pi_{\text {rig }}: D_{\beta_{i}} \rightarrow D_{\alpha_{i}}$ admits a section on $D_{\alpha_{i}}(0, e /(e+1))$ whose image is $D_{\beta_{i}}(0, e /(e+1))$. The map $\pi_{\text {rig }}: D_{\beta_{i}} \rightarrow$ $D_{\alpha_{i}}$ is the analytification of the $\operatorname{map} \pi: \operatorname{Spf}\left(\mathcal{O}_{Y}^{\wedge \beta_{i}}\right) \rightarrow \operatorname{Spf}\left(\mathcal{O}_{X}^{\wedge \alpha_{i}}\right)$ by Proposition 2.2. By Lemma 2.4, choosing local coordinates, this map is given by

$$
\mathcal{O} \llbracket t \rrbracket \rightarrow \mathcal{O} \llbracket x, y \rrbracket /(x y-\varpi), \quad t \mapsto x+u y^{e}+f(y)+\varpi g,
$$

where $f(y) \equiv 0\left(\bmod y^{e+1}\right), u, g \in \mathcal{O} \llbracket x, y \rrbracket /(x y-\varpi)$, and $u$ is a unit. Let $\tilde{u}, \tilde{g}$ denote arbitrary liftings of $u, g$ to $\mathcal{O} \llbracket x, y \rrbracket$ and define $g_{0}(x)=\tilde{g}(x, \varpi / x), u_{0}(x)=$ $\tilde{u}(x, \varpi / x)$, and $f_{0}(x)=f(\varpi / x)$. Then the map $\pi_{\text {rig }}: D_{\beta_{i}} \rightarrow D_{\alpha_{i}}$ is the map characterized by

$$
t\left(\pi_{\text {rig }} Q\right)=x(Q)+u_{0}(x(Q))(\varpi / x(Q))^{e}+f_{0}(x(Q))+\varpi g_{0}(x(Q)) .
$$


Lemma 3.6. Let $Q \in \mathfrak{Y}_{\text {rig. }}$.

(1) If $\nu_{\mathfrak{Y}}(Q)<e /(e+1)$ then $v_{\mathfrak{X}}\left(\pi_{\text {rig }} Q\right)=v_{\mathfrak{Y}}(Q)$.

(2) If $\nu_{\mathfrak{Y}}(Q)>e /(e+1)$ then $\nu_{\mathfrak{X}}\left(\pi_{\text {rig }} Q\right)=e\left(1-\nu_{\mathfrak{Y}}(Q)\right)<e /(e+1)$.

(3) If $\nu_{\mathfrak{Y}}(Q)=e /(e+1)$ then $\nu_{\mathfrak{X}}\left(\pi_{\mathrm{rig}} Q\right) \geq e /(e+1)$.

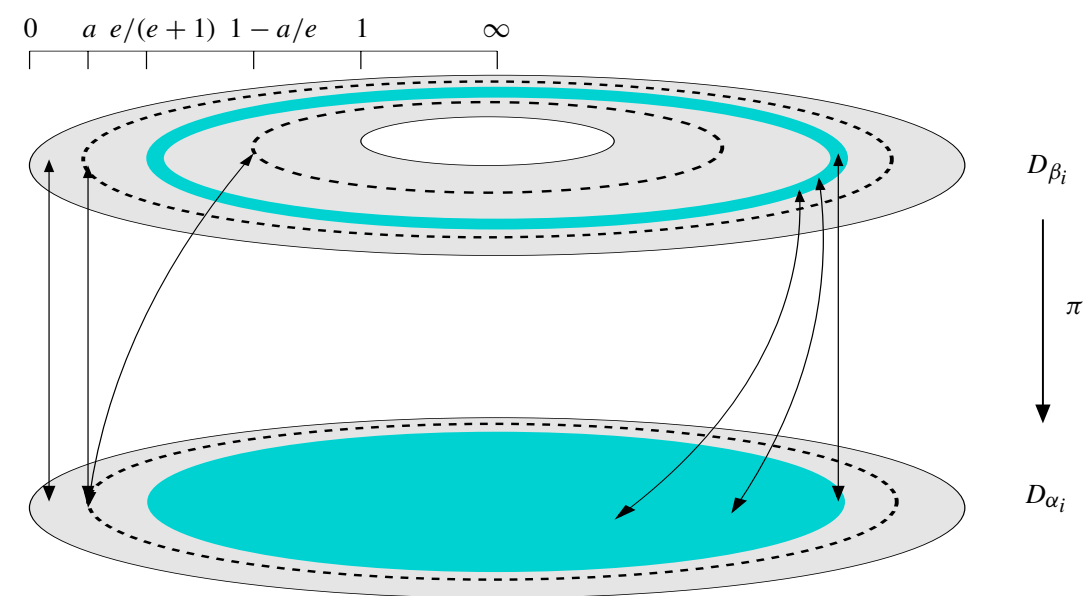

Figure 3.1. The effect of $\pi_{\text {rig }}$ on measures of singularity.

Proof. The statement is clear for $Q \in \mathscr{Z}^{\infty} \cup \mathscr{Z}^{0}$. If $Q \in D_{\beta_{i}}$ satisfies $\nu_{\mathfrak{Y}}(Q)=$ $\operatorname{val}(x(Q))<e /(e+1)$, then

$$
\operatorname{val}(x(Q))<\min \left\{\operatorname{val}\left((\varpi / x(Q))^{e}\right), \operatorname{val}\left(f_{0}(x(Q))\right), \operatorname{val}\left(\varpi g_{0}(x(Q))\right)\right\} .
$$

This implies that $\operatorname{val}\left(t\left(\pi_{\text {rig }} Q\right)\right)=\operatorname{val}(x(Q))$. The other cases are similar.

From the lemma it follows that

$$
\pi_{\mathrm{rig}}{ }^{-1} D_{\alpha_{i}}(0, e /(e+1))=D_{\beta_{i}}(0, e /(e+1)) \coprod D_{\beta_{i}}(e /(e+1), 1) .
$$

Indeed the lemma proves something stronger: for any $a \in \mathbb{Q}$ satisfying $0<a<$ $e /(e+1)$ we have

$$
\pi_{\mathrm{rig}}{ }^{-1}\left(D_{\alpha_{i}}[a, a]\right)=D_{\beta_{i}}[1-a / e, 1-a / e] \cup D_{\beta_{i}}[a, a] .
$$

This shows that the inverse image of $D_{\alpha_{i}}(0, e /(e+1))$ under $\pi_{\text {rig }}$ has two connected components each of which maps onto $D_{\alpha_{i}}(0, e /(e+1))$ in a finite flat manner.

We show that the finite flat morphism $\pi_{\text {rig }}: D_{\beta_{i}}(0, e /(e+1)) \rightarrow D_{\alpha_{i}}(0, e /(e+1))$ is of degree one and hence is an isomorphism. The inverse of this map provides the 
desired section $t$. To calculate the degree we restrict the map to a circle $D_{\alpha_{i}}[a, a]$ with $0<a<e /(e+1)$. It is therefore enough to show that $\pi_{\text {rig }}: D_{\beta_{i}}[a, a] \rightarrow D_{\alpha_{i}}[a, a]$ has degree one. We show this by reduction modulo $\varpi$. Our argument is based on the following general principle.

Let $\phi: \operatorname{Spm}(B) \rightarrow \operatorname{Spm}(A)$ be a finite flat morphism of $K$-affinoids. Let $L$ be a finite field extension of $K$ and let $\phi_{L}: \operatorname{Spm}\left(B \otimes_{K} L\right) \rightarrow \operatorname{Spm}\left(A \otimes_{K} L\right)$ be the induced morphism. Let $\theta$ be a uniformizer of $L$ and let $n$ be a positive integer; let $\left(B \otimes_{K} L\right)^{\circ}$ denote the $\mathcal{O}_{L}$-algebra of functions of supremum norm at most 1 . Define $\bar{B}=\left(B \otimes_{K} L\right)^{\circ} /\left(\theta^{n}\right)$, and similarly for $A$. Let $\bar{\phi}_{L}: \operatorname{Spec}(\bar{B}) \rightarrow \operatorname{Spec}(\bar{A})$ be the induced map. Then, if $\bar{\phi}_{L}$ is an isomorphism so is $\phi$. The argument reduces to proving that $\phi_{L}^{*}:\left(A \otimes_{K} L\right)^{\circ} \rightarrow\left(B \otimes_{K} L\right)^{\circ}$ is surjective, which, in turn, follows from Nakayama's lemma.

To prove that the reduction of $\pi_{\text {rig }}: D_{\beta_{i}}[a, a] \rightarrow D_{\alpha_{i}}[a, a]$ is an isomorphism, we first re-scale. We pass to a finite extension $L$ of $K$ with uniformizer $\theta$ in which there exists an element $\lambda$ of valuation $a$. Setting $x=\lambda x_{0}$ and $t=\lambda t_{0}$ the map $\pi_{\text {rig }}$ becomes a map between circles of radius one characterized by

$$
\begin{aligned}
t_{0}\left(\pi_{\text {rig }} Q\right)= & x_{0}(Q)+u_{0}\left(\lambda x_{0}(Q)\right)\left(\varpi^{e} / \lambda^{e+1}\right) x_{0}(Q)^{-e} \\
& +\lambda^{-1} f_{0}\left(\lambda x_{0}(Q)\right)+\lambda^{-1} \varpi g_{0}\left(\lambda x_{0}(Q)\right) .
\end{aligned}
$$

Using $0<a<e(e+1)^{-1}$ and $f(y) \equiv 0\left(\bmod y^{e+1}\right)$, one sees that this map reduces modulo $\theta$ to the identity map of $\mathcal{O}_{L} /(\theta)[T, 1 / T]$.

For the second statement we argue as follows. Let $U \supseteq D_{\alpha_{i}}[a, a]$ be a connected admissible open of $\mathfrak{X}_{\text {rig }}(0, e /(e+1))$ over which there is a section $\mathfrak{t}^{\prime}$ to $\pi_{\text {rig. Then }}$ $U$, being connected, lies entirely within $D_{\alpha_{i}}(0, e /(e+1))$. By Lemma 3.6 the image of $U$ under $\mathfrak{t}^{\prime}$ is either a subset of $D_{\beta_{i}}(0, e /(e+1))$, or a subset of $D_{\beta_{i}}(e /(e+1), 1)$. In the former case, by the construction of $\mathfrak{t}$, it is clear that $\mathfrak{t}^{\prime}=\left.\mathfrak{t}\right|_{U}$. In the latter case, $\mathfrak{t}^{\prime}\left(D_{\alpha_{i}}[a, a]\right)$ is a connected component of $D_{\beta_{i}}[1-a / e, 1-a / e]$ by Equation (3.1). However, since $D_{\beta_{i}}[1-a / e, 1-a / e]$ is connected and $\pi_{\text {rig }}: D_{\beta_{i}}[1-a / e, 1-a / e] \rightarrow$ $D_{\alpha_{i}}[a, a]$ is $e$-to- 1 , we find that $e=1$, which contradicts our assumption.

Corollary 3.7. Assume $e>1$. The morphism $\pi_{\text {rig }}: \mathfrak{Y}_{\text {rig }} \rightarrow \mathfrak{X}_{\text {rig }}$ admits a unique section

$$
\mathfrak{s}_{\text {rig }}: \mathfrak{X}_{\text {rig }}[0, e /(e+1)) \rightarrow \mathfrak{Y}_{\text {rig }}
$$

which extends $\mathfrak{s}_{\text {rig }}^{\infty}$.

Proof. By Corollary $3.4, \mathfrak{s}_{\text {rig }}^{\infty}$ extends to a section $\mathfrak{s}_{\text {rig }}^{\dagger}$ on $\mathfrak{X}_{\text {rig }}[0, a]$ for some positive rational number $a<e /(e+1)$. By the uniqueness assertion in Proposition 3.5 we know that the restriction of $\mathfrak{s}_{\text {rig }}^{\dagger}$ to $\mathfrak{X}_{\text {rig }}(0, a]$ is obtained as the restriction of $\mathfrak{t}$. This implies that $\mathfrak{s}_{\text {rig }}^{\infty}$ and $\mathfrak{t}$ glue together to form the desired unique section. 
Proposition 3.8. Assume $e>1$. The section $\mathfrak{s}_{\text {rig }}$ constructed in Corollary 3.7 is maximal in the following sense: Let $U$ be a connected affinoid inside $\mathfrak{X}_{\mathrm{rig}}(0, \infty)$ such that it intersects both $\mathfrak{X}_{\mathrm{rig}}(0, e /(e+1))$ and $\mathfrak{X}_{\mathrm{rig}}[e /(e+1), 1)$ nontrivially . Then there is no section to $\pi_{\text {rig }}$ on $U$.

Proof. Assume there is a section $\mathfrak{s}$ to $\pi_{\text {rig }}$ on such $U$. As $U$ is connected it lies inside some $D_{\alpha_{i}}$, and intersects both $D_{\alpha_{i}}(0, e /(e+1))$ and $D_{\alpha_{i}}[e /(e+1), e /(e+1)]$ nontrivially. By [BGR, §9.7.2, Thm. 2] any connected affinoid of $D_{\alpha_{i}}$ is the complement of a union of finitely many disjoint open discs in a closed disc. A simple calculation using the non-archimedean property of the norm shows that a closed disc which intersects both $D_{\alpha_{i}}(0, e /(e+1))$ and $D_{\alpha_{i}}[e /(e+1), e /(e+1)]$ nontrivially, must contain all of $D_{\alpha_{i}}[e /(e+1), e /(e+1)]$. Therefore, $U$ contains the complement of a union of finitely many disjoint open discs $V_{1}, \ldots, V_{r}$ (which we may assume to have radius $e /(e+1))$ in the circle $D_{\alpha_{i}}[e /(e+1), e /(e+1)]$.

We first re-scale as in the proof of Proposition 3.5: let $\lambda \in L$ be such that $\operatorname{val}(\lambda)=$ $e /(e+1)$. Setting $x=\lambda x_{0}$ and $t=\lambda t_{0}$ the map $\pi_{\text {rig }}: D_{\beta_{i}}[e /(e+1), e /(e+1)] \rightarrow$ $D_{\alpha_{i}}[e /(e+1), \infty)$ becomes a map between a circle $C$ of radius one and the closed unit disc $D$ characterized by

$$
\begin{aligned}
t_{0}\left(\pi_{\text {rig }} Q\right)= & x_{0}(Q)+u_{0}\left(\lambda x_{0}(Q)\right)\left(\varpi^{e} / \lambda^{e+1}\right) x_{0}(Q)^{-e} \\
& +\lambda^{-1} f_{0}\left(\lambda x_{0}(Q)\right)+\lambda^{-1} \varpi g_{0}\left(\lambda x_{0}(Q)\right) .
\end{aligned}
$$

The section $\mathfrak{s}$ is defined on $W$, the complement in $D$ of finitely many residue discs which are the open unit disc together with $\lambda^{-1} V_{1}, \ldots, \lambda^{-1} V_{r}$. The reduction $\overline{\mathfrak{s}}$ of $\mathfrak{s}: W \rightarrow C$, then, gives a map between $\mathbb{A}_{\mathcal{O}_{L} /(\theta)}^{1}$ minus a finite number of points (with parameter $\bar{t}_{0}$ ), and $\mathbb{A}_{\mathcal{O}_{L} /(\theta)}^{1}$ (with parameter $\bar{x}_{0}$ ) characterized by

$$
\bar{t}_{0}(Q)=\bar{x}_{0}(\overline{\mathfrak{s}}(Q))+\overline{\left(\varpi^{e} / \lambda^{e+1}\right)} \bar{x}_{0}(\overline{\mathfrak{s}}(Q))^{-e} .
$$

Here $\bar{x}_{0}(\overline{\mathfrak{s}}(-))$ is a rational function in $\bar{t}_{0}$ and $\overline{\varpi^{e} / \lambda^{e+1}}$ is nonzero by our choice of $\lambda$. Degree considerations show that this is impossible.

We summarize the above results as a theorem (Theorem A of the Introduction).

Theorem 3.9. Assume $e>1$. The morphism $\pi_{\text {rig }}: \mathfrak{Y}_{\text {rig }} \rightarrow \mathfrak{X}_{\text {rig }}$ admits a section

$$
\mathfrak{s}_{\text {rig }}: \mathfrak{X}_{\text {rig }}[0, e /(e+1)) \rightarrow \mathfrak{Y}_{\text {rig }} .
$$

This section is maximal, namely, it can not be extended to any connected admissible open set properly containing $\mathfrak{X}_{\text {rig }}[0, e /(e+1))$.

The canonical subgroup of an elliptic curve can be thought of as a certain lifting of the kernel of Frobenius from characteristic $p$ [Kat, Thm. 3.1]. We prove a similar 
result in our setting. The section $\mathfrak{s}_{\text {rig }}^{\infty}=\left.\mathfrak{s}_{\text {rig }}\right|_{\mathscr{Z}}$ was constructed on the level of formal schemes, and by its construction it reduces to $s \bmod \varpi$.

Fix $D_{\alpha_{i}}$ and let $t$ be a coordinate on it, obtained from an isomorphism $\mathcal{O}_{X}^{\wedge \alpha_{i}} \cong$ $\mathcal{O} \llbracket t \rrbracket$ as in Lemma 2.4. Also fix an isomorphism $\mathcal{O}_{Y}^{\wedge \beta_{i}} \cong \mathcal{O} \llbracket x, y \rrbracket /(x y-\varpi)$ as in loc. cit.; $x$ is a parameter on $D_{\beta_{i}}$ and $x y=\varpi$.

Let $\gamma_{P}: \operatorname{Spm}(L) \rightarrow D_{\alpha_{i}}$ correspond to a closed point $P$ which is contained in $D_{\alpha_{i}}(0, e /(e+1))$. Thus $L$ is a finite extension of $K$. Let $\gamma_{\mathfrak{s}_{\text {rig }}(P)}=\mathfrak{s}_{\text {rig }} \circ$ $\gamma_{P}: \operatorname{Spm}(L) \rightarrow D_{\beta_{i}}$ correspond to the image of $P$ under $\mathfrak{s}_{\text {rig. }}$. Let $\tilde{\gamma}_{P}: \operatorname{Spf}\left(\mathcal{O}_{L}\right) \rightarrow \mathfrak{X}$ denote the extension of $\gamma_{P}$ to the formal model, and similarly define $\tilde{\gamma}_{\mathfrak{r}_{\text {rig }}(P) \text {. Let }}$ $\bar{\gamma}_{P}, \bar{\gamma}_{\mathfrak{s}_{\text {rig }}(P)}$ denote, respectively, the reductions of $\tilde{\gamma}_{P}, \tilde{\gamma}_{\mathfrak{s}_{\mathrm{rig}}(P)}$ modulo the element $\varpi / t(P)$ of $\mathcal{O}_{L}$. Let $s^{\prime}$ denote the base change of $s: X \otimes \kappa \rightarrow Y \otimes \kappa$ from $\kappa$ to $\mathcal{O}_{L} /(\varpi / t(P))$. For simplicity we denote the $\kappa$-algebra $\mathcal{O}_{L} /(\varpi / t(P))$ by $R$.

Proposition 3.10. For closed points $P \in D_{\alpha_{i}}(0, e /(e+1))$ with $t(P)=r \in \mathcal{O}_{L}$ the section $\mathfrak{s}_{\text {rig }}$ reduces modulo $\varpi / r$ to $s^{\prime}$. More precisely, for any $P \in D_{\alpha_{i}}(0, e /(e+1))$ we have $\bar{\gamma}_{\mathfrak{s}_{\mathrm{rig}}(P)}=s^{\prime} \circ \bar{\gamma}_{P}$.

Proof. Let us denote the image of an element $a \in \mathcal{O}_{L}$ in $R:=\mathcal{O}_{L} /(\varpi / t(P))$ by $\bar{a}$. Since $P \in D_{\alpha_{i}}$, the map $\tilde{\gamma}_{P}: \operatorname{Spf}\left(\mathcal{O}_{L}\right) \rightarrow \mathfrak{X}$ factors through $\operatorname{Spf}\left(\mathcal{O}_{X}^{\wedge \alpha_{i}}\right)$. Similarly $\tilde{\gamma}_{\mathfrak{s}_{\text {rig }}(P)}$ factors through $\operatorname{Spf}\left(\mathcal{O}_{Y}^{\wedge \beta_{i}}\right)$. Therefore, it is enough to prove the statement after replacing $\mathfrak{X}$ with $\operatorname{Spf}\left(\mathcal{O}_{X}^{\wedge \alpha_{i}}\right)$ and $\mathfrak{Y}$ with $\operatorname{Spf}\left(\mathcal{O}_{Y}^{\wedge \beta_{i}}\right)$. Then

$$
\bar{\gamma}_{P}: \operatorname{Spec}(R) \rightarrow \operatorname{Spec}\left(\mathcal{O}_{X}^{\wedge \alpha_{i}} \otimes R\right) \cong \operatorname{Spec}(R \llbracket t \rrbracket)
$$

is given by $t \mapsto \overline{t(P)}$. Similarly, the map

$$
\bar{\gamma}_{\mathfrak{s}_{\text {rig }}(P)}: \operatorname{Spec}(R) \rightarrow \operatorname{Spec}\left(\mathcal{O}_{Y}^{\wedge \beta_{i}} \otimes R\right) \cong \operatorname{Spec}(R \llbracket x, y \rrbracket /(x y))
$$

is given by $x \mapsto \overline{x\left(\mathfrak{s}_{\text {rig }}(P)\right)}, y \mapsto \overline{y\left(\mathfrak{s}_{\text {rig }}(P)\right)}$. From the proof of Lemma 2.4, we see that the section

$$
\begin{aligned}
s^{\prime}: \operatorname{Spec}(R \llbracket t \rrbracket) & \cong \operatorname{Spec}\left(\mathcal{O}_{X}^{\wedge \alpha_{i}} \otimes \kappa \otimes_{\kappa} R\right) \rightarrow \operatorname{Spec}\left(\mathcal{O}_{Y}^{\wedge \beta_{i}} \otimes \kappa \otimes_{\kappa} R\right) \\
& \cong \operatorname{Spec}(R \llbracket x, y \rrbracket /(x y))
\end{aligned}
$$

is given by $x \mapsto t, y \mapsto 0$. Hence, it is enough to show that $\overline{y\left(\mathfrak{s}_{\text {rig }}(P)\right)}=0$ and $\overline{x\left(\mathfrak{s}_{\text {rig }}(P)\right)}=\overline{t(P)}$. For the first equality notice that by Lemma 3.6 we have $\operatorname{val}(t(P))=\operatorname{val}\left(x\left(\mathfrak{s}_{\text {rig }}(P)\right)\right)$ and hence $y\left(\mathfrak{s}_{\text {rig }}(P)\right)=\varpi / x\left(\mathfrak{s}_{\text {rig }}(P)\right)$ is divisible by $\varpi / t(P)$. Since $\operatorname{val}(t(P))=\operatorname{val}\left(x\left(\mathfrak{s}_{\text {rig }}(P)\right)\right)$, to prove the second equality it is enough to show that $t(P)$ and $x\left(\mathfrak{s}_{\text {rig }}(P)\right)$ have the same reduction modulo $y\left(\mathfrak{s}_{\text {rig }}(P)\right)=$ $\varpi / x\left(\mathfrak{s}_{\text {rig }}(P)\right)$. But that is clear since from the proof of Proposition 3.5 we have

$$
\begin{aligned}
t(P)=x & \left(\mathfrak{s}_{\text {rig }}(P)\right)+u_{0}\left(x\left(\mathfrak{s}_{\text {rig }}(P)\right)\right)\left(y\left(\mathfrak{s}_{\text {rig }}(P)\right)\right)^{e} \\
& +f_{0}\left(x\left(\mathfrak{s}_{\text {rig }}(P)\right)\right)+\varpi g_{0}\left(x\left(\mathfrak{s}_{\text {rig }}(P)\right)\right),
\end{aligned}
$$


and $f_{0}\left(x\left(\mathfrak{s}_{\text {rig }}(P)\right)\right) \equiv 0\left(\bmod y\left(\mathfrak{s}_{\text {rig }}(P)\right)^{e+1}\right)$.

Definition 3.11. Let $Q \in \mathfrak{Y}_{\text {rig }}$.

(1) We say that $Q$ is canonical if $Q$ is in the image of $\mathfrak{s}_{\text {rig. }}$. By the construction of $\mathfrak{s}_{\text {rig }}$, this is equivalent to having $\nu_{\mathfrak{Y}}(Q)<e /(e+1)$. If $Q$ is canonical, then by Lemma 3.6 we have $v_{\mathfrak{Y}}(Q)=v_{\mathfrak{X}}\left(\pi_{\text {rig }} Q\right)$.

(2) We say that $Q$ is anti-canonical if $\nu_{\mathfrak{Y}}(Q)>e /(e+1)$. In this case by Lemma 3.6 we have $\nu_{\mathfrak{Y}}(Q)=1-e^{-1} \nu_{\mathfrak{X}}\left(\pi_{\text {rig }} Q\right)$.

(3) We say that $Q$ is too singular if $\nu_{\mathfrak{Y}}(Q)=e /(e+1)$. This is equivalent to $v_{\mathfrak{X}}\left(\pi_{\text {rig }} Q\right) \geq e /(e+1)$.

Remark 3.12. In the context of modular curves, the measure of singularity $\nu_{\mathfrak{Y}}$ was first introduced by Buzzard in $\S 4$ of [Buz2]. In [Co] this measure, referred to as the Buzzard invariant, was used to identify "circles" corresponding to the image in $X_{0}(p)$ of points on $X_{0}\left(p^{2}\right)$ whose reductions lie on the horizontal components of the special fibre of Edixhoven's stable model of $X_{0}\left(p^{2}\right)$. As it was pointed to us by one of the referees, the setting in this work seems suitable for carrying out Coleman's approach (which is less explicit than this work, but contains observations in the same spirit), and hence clarifying how his results can be extended to the case of non-trivial tame level and to corresponding situations for other Shimura varieties.

\section{Throwing in an "involution"}

In this section we prove the following theorem (Theorem B of the Introduction).

Theorem 4.1. Let $w$ be an automorphism of $\mathfrak{Y}$ that permutes the components of $\mathfrak{Y}$. We denote by $w$ also the induced automorphism of $\mathfrak{Y}_{\text {rig }}$ and its effect of points by $Q \mapsto Q^{w}$. Then:

(1) $v_{\mathfrak{X}}\left(\pi_{\text {rig }} Q\right)=0 \Leftrightarrow v_{\mathfrak{X}}\left(\pi_{\text {rig }} Q^{w}\right)=0$. In this case $Q$ is canonical if and only if $Q^{w}$ is anti-canonical.

(2) If $\nu_{\mathfrak{X}}\left(\pi_{\text {rig }} Q\right)<(e+1)^{-1}$ and $Q$ canonical, then $\nu_{\mathfrak{X}}\left(\pi_{\text {rig }} Q^{w}\right)=e \cdot v_{\mathfrak{X}}\left(\pi_{\text {rig }} Q\right)$ and $Q^{w}$ is anti-canonical.

(3) If $\nu_{\mathfrak{X}}\left(\pi_{\mathrm{rig}} Q\right)=(e+1)^{-1}$, and $Q$ is canonical, then $Q^{w}$ is too singular.

(4) If $(e+1)^{-1}<v_{\mathfrak{X}}\left(\pi_{\text {rig }} Q\right)<e(e+1)^{-1}$, and $Q$ is canonical, then $v_{\mathfrak{X}}\left(\pi_{\text {rig }} Q^{w}\right)=$ $1-v_{\mathfrak{X}}\left(\pi_{\mathrm{rig}} Q\right)$ and $Q^{w}$ is canonical.

(5) If $v_{\mathfrak{X}}\left(\pi_{\mathrm{rig}} Q\right)<e(e+1)^{-1}$, and $Q$ is anti-canonical, then $v_{\mathfrak{X}}\left(\pi_{\mathrm{rig}} Q^{w}\right)=$ $e^{-1} v_{\mathfrak{X}}\left(\pi_{\mathrm{rig}} Q\right)$, and $Q^{w}$ is canonical.

(6) If $Q$ is too singular, then $\nu_{\mathfrak{X}}\left(\pi_{\mathrm{rig}} Q^{w}\right)=(e+1)^{-1}$ and $Q^{w}$ is canonical. 
We begin by proving the following lemma.

Lemma 4.2. For any $Q \in \mathfrak{Y}_{\text {rig }}$ we have

$$
\nu_{\mathfrak{Y}}(Q)+\nu_{\mathfrak{Y}}\left(Q^{w}\right)=1 .
$$

Proof. We first note that $w\left(\mathscr{Z}^{\infty}\right)=\mathscr{Z}^{0}$, and hence for $Q \in \mathscr{Z}^{\infty} \cup \mathscr{Z}^{0}$ the result follows from the definition of $\nu_{\mathfrak{Y}}$. Assume $Q \in D_{\beta_{i}}$ for some $1 \leq i \leq h$. The automorphism $w$ induces an isomorphism between $D_{\beta_{i}}$ and $D_{\beta_{j}}$ where $\beta_{j}=\beta_{i}^{w}$. Let $x, y$ be coordinates on $D_{\beta_{i}}$ as in Lemma 2.4. Then $\eta:=w^{*} x$ and $\xi:=w^{*} y$ are coordinates on $D_{\beta_{j}}$ such that $D_{\beta_{j}}$ is the analytification of $\operatorname{Spf}(\mathcal{O} \llbracket \xi, \eta \rrbracket /(\xi \eta-$ $\varpi)$ ). Because $w$ switches the two components of $Y \otimes \kappa, \xi$ is a local parameter on the component containing $(Y \otimes \kappa)^{\infty}$ at the point $\beta_{j}$. Examination of the proof of Lemma 2.4 shows that there is a local parameter $\tau$ on $D_{\alpha_{j}}$, and local parameters $(\hat{\xi}, \hat{\eta})$ on $D_{\beta_{j}}$ such that $\hat{\xi}=\xi \hat{u}, \hat{\eta}=\eta \hat{u}^{-1}$, where $\hat{u}$ is a unit in $\mathcal{O} \llbracket \xi, \eta \rrbracket /(\xi \eta-\varpi)$, and such that $(\tau, \hat{\xi}, \hat{\eta})$ are related as in the statement of Lemma 2.4.

By our definition, we can use $\hat{\xi}$ to calculate $\nu_{\mathfrak{Y}}$ on $D_{\beta_{j}}$. Therefore

$$
\begin{aligned}
\nu_{\mathfrak{Y}}\left(Q^{w}\right) & =\operatorname{val}\left(\hat{\xi}\left(Q^{w}\right)\right)=\operatorname{val}\left(\xi\left(Q^{w}\right)\right) \\
& =\operatorname{val}(y(Q))=1-\operatorname{val}(x(Q))=1-\nu_{\mathfrak{Y}}(Q) .
\end{aligned}
$$

We now prove the theorem.

(1) is clear.

(2) As $Q$ is canonical, Lemma 3.6 implies that $v_{\mathfrak{Y}}(Q)=v_{\mathfrak{X}}\left(\pi_{\text {rig }} Q\right)<(e+1)^{-1}$. Therefore by Lemma 4.2 we have $\nu_{\mathfrak{Y}}\left(Q^{w}\right)>e(e+1)^{-1}$, which means that $Q^{w}$ is anti-canonical. It now follows from Lemma 3.6 that $\nu_{\mathfrak{X}}\left(\pi_{\text {rig }} Q^{w}\right)=e\left(1-\nu_{\mathfrak{Y}}\left(Q^{w}\right)\right)=$ $e \nu_{\mathfrak{Y}}(Q)=e \nu_{\mathfrak{X}}\left(\pi_{\text {rig }} Q\right)$.

(3) As $Q$ is canonical, Lemma 3.6 implies that $\nu_{\mathfrak{Y}}(Q)=v_{\mathfrak{X}}\left(\pi_{\text {rig }} Q\right)=(e+1)^{-1}$, and therefore $\nu_{\mathfrak{Y}}\left(Q^{w}\right)=e(e+1)^{-1}$. This shows that $Q^{w}$ is too singular. It follows from Lemma 3.6 that $\nu_{\mathfrak{X}}\left(\pi_{\text {rig }} Q^{w}\right) \geq e(e+1)^{-1}$.

(4) Since $Q$ is canonical, we have $\nu_{\mathfrak{Y}}(Q)=v_{\mathfrak{X}}\left(\pi_{\text {rig }} Q\right)>(e+1)^{-1}$, and hence $\nu_{\mathfrak{Y}}\left(Q^{w}\right)<e(e+1)^{-1}$. This shows that $Q^{w}$ is canonical. Therefore, $\nu_{\mathfrak{X}}\left(\pi_{\mathrm{rig}} Q^{w}\right)=$ $v_{\mathfrak{Y}}\left(Q^{w}\right)=1-v_{\mathfrak{Y}}(Q)=1-v_{\mathfrak{X}}\left(\pi_{\text {rig }} Q\right)$.

(5) Since $Q$ is anti-canonical, Lemma 3.6 shows that $\nu_{\mathfrak{Y}}(Q)=1-e^{-1} v_{\mathfrak{X}}\left(\pi_{\text {rig }} Q\right)>$ $e(e+1)^{-1}$. Therefore, $v_{\mathfrak{Y}}\left(Q^{w}\right)=1-v_{\mathfrak{Y}}(Q)<(e+1)^{-1}$ and hence $Q^{w}$ is canonical. We have $\nu_{\mathfrak{X}}\left(\pi_{\text {rig }} Q^{w}\right)=\nu_{\mathfrak{Y}}\left(Q^{w}\right)=1-v_{\mathfrak{Y}}(Q)=e^{-1} \nu_{\mathfrak{X}}\left(\pi_{\text {rig }} Q\right)$.

(6) Since $\nu_{\mathfrak{X}}\left(\pi_{\text {rig }} Q\right) \geq e(e+1)^{-1}$, by Lemma 3.6 we have $\nu_{\mathfrak{Y}}(Q)=e(e+1)^{-1}$, and hence $\nu_{\mathfrak{Y}}\left(Q^{w}\right)=(e+1)^{-1}$, This shows that $Q^{w}$ is canonical. Therefore, we have $v_{\mathfrak{X}}\left(\pi_{\text {rig }} Q^{w}\right)=v_{\mathfrak{Y}}\left(Q^{w}\right)=(e+1)^{-1}$. 


\section{Applications}

In this section we review some of the structure theory for Shimura curves and show that our results apply to these situations. Our main references are Drinfeld [Dri1], [Dri2] and Carayol [Car]. In particular, we reproduce the classical results on canonical subgroups [Kat], as well as more recent developments [Kas1], [Kas2].

Let $F$ be a totally real field of degree $d$ with ring of integers $\mathcal{O}_{F}$ and let $B / F$ be a quaternion algebra split at exactly one infinite prime of $F$. Let $R$ be a maximal order of $B$. Let $\mathfrak{p}$ be a finite prime of $F$ at which $B$ splits, $F_{\mathfrak{p}}$ the completion of $F$ at the prime $\mathfrak{p}, \mathcal{O}_{F, \mathfrak{p}}$ its ring of integers with a uniformizer $\varpi$, and identify $B \otimes_{F} F_{\mathfrak{p}}$ with $M_{2}\left(F_{\mathfrak{p}}\right)$ so that $R \otimes_{\mathcal{O}_{F}} \mathcal{O}_{F, \mathfrak{p}}=M_{2}\left(\mathcal{O}_{F, \mathfrak{p}}\right)$. With $B$ there is associated a projective system of Shimura curves, initially over the complex numbers but, by Shimura's theory of canonical models, in fact over $F$. Let $G=\operatorname{Res}_{F / \mathbb{Q}}\left(B^{\times}\right)$. Let $X$ be the $G(\mathbb{R})$-conjugacy class of the homomorphism $\mathbb{C}^{\times} \rightarrow G(\mathbb{R})$ sending $x+i y$ to $\left[\left(\begin{array}{cc}x & y \\ -y & x\end{array}\right), 1, \ldots, 1\right] \in \mathrm{GL}_{2}(\mathbb{R}) \times\left(\mathbb{H}^{\times}\right)^{d-1}$. Let $K$ be an open compact subgroup of $G\left(\mathbb{A}^{f}\right)$ of the form $K_{\mathfrak{p}} \times K^{\mathfrak{p}}$, where $K_{\mathfrak{p}} \subseteq \mathrm{GL}_{2}\left(\mathcal{O}_{F, \mathfrak{p}}\right)$ and $K^{\mathfrak{p}}$ is "away from $\mathfrak{p}$ ". The Shimura curve associated with $K$ is $M_{K}(G, X)(\mathbb{C})=G(\mathbb{Q}) \backslash G\left(\mathbb{A}^{f}\right) \times X / K$.

5.1. The case $\boldsymbol{F}=\mathbb{Q}$. In this case the Shimura curves $M_{K}(G, X) / \mathbb{Q}$ afford a natural modular description. Consider the functor associating to a scheme $S$ the isomorphism classes of triples $(A, \iota, \alpha) / S$, where $A / S$ is an abelian scheme of relative dimension 2, $\iota: R \rightarrow \operatorname{End}_{S}(A)$ is an injective ring homomorphism and $\alpha: R / N R \rightarrow A[N]$ is an isomorphism of $R$-group schemes; c.f. [Dri2, §4], [DT, §4], [Buz1]. (Such objects are sometimes called "false elliptic curves" because of the similarity with the case of $B=M_{2}(\mathbb{Q})$ and the usual modular curves.) This corresponds to the case where $K$ is $\Gamma(N)$ - the elements of $\left(R \otimes_{\mathbb{Z}} \widehat{\mathbb{Z}}\right)^{\times}$(viewed as a subgroup of $\left.G\left(\mathbb{A}^{f}\right)\right)$ that reduce to the identity element under $\left(R \otimes_{\mathbb{Z}} \widehat{\mathbb{Z}}\right)^{\times} \rightarrow\left(R \otimes_{\mathbb{Z}} \mathbb{Z} / N \mathbb{Z}\right)^{\times}$. For a general $K, K$ contains $\Gamma(N)$ for some $N$ and we take $\alpha$ up to $K$-equivalence (étale locally). This makes sense in all characteristics once the level structure is understood in Drinfeld's sense for which we refer to [Dri1], [KM]. For $K$ small enough, there is therefore a scheme $\mathbf{M}_{K}$ over $\operatorname{Spec}(\mathbb{Z})$ representing this functor such that $\mathbf{M}_{K} \otimes_{\mathbb{Z}} \mathbb{Q} \cong M_{K}(G, X)$.

As a module over $R \otimes \mathbb{Z}_{p}=M_{2}\left(\mathbb{Z}_{p}\right)$, the $p$-divisible group $A\left[p^{\infty}\right]$ of $A / S$ is a direct sum $A\left[p^{\infty}\right]_{1} \oplus A\left[p^{\infty}\right]_{2}$ of two isomorphic $p$-divisible groups over $S$, where the decomposition is determined by the orthogonal idempotents $\left(\begin{array}{ll}1 & 0 \\ 0 & 0\end{array}\right)$ and $\left(\begin{array}{ll}0 & 0 \\ 0 & 1\end{array}\right)$ in $M_{2}\left(\mathbb{Z}_{p}\right)$; furthermore, these idempotents are conjugate under $\left(\begin{array}{ll}0 & 1 \\ 1 & 0\end{array}\right)$, which induces the isomorphism $A\left[p^{\infty}\right]_{1} \cong A\left[p^{\infty}\right]_{2}$. Let $K^{p}$ be small enough and let $K_{p}$ be the standard Iwahori subgroup of $\mathrm{GL}_{2}\left(\mathbb{Z}_{p}\right)$. The open compact subgroup $K=K_{p} \times K^{p}$ corresponds to a choice of level structure away from $p$ (given by $K^{p}$ ) and a choice of a non-trivial ideal $H \subset M_{2}(\mathbb{Z} / p \mathbb{Z})$. Such $H$ corresponds, via the $K_{p}$-equivalence class of $\alpha$, to an $R$-invariant subgroup of $A[p]$ of degree $p^{2}$. The level structure at $p$ can therefore also be expressed as an isogeny $f: A_{1} \rightarrow A_{2}$ of false elliptic curves 
whose kernel is of degree $p^{2}$ and is killed by $p$. The conditions on $f$ can also be formulated by requiring $f$ to have "false degree" $p$, i.e. that $f^{t} \circ f=[p]$ (see below for the exact meaning of this formula); c.f. [DT, p. 453], [Kas1, §§10-11].

Let $A / k$ be a false elliptic curve over an algebraically closed field $k$ of characteristic $p$. One can prove, by means of the idempotents we have chosen, that the functor of infinitesimal deformations of $A$ (resp., together with an Iwahori level structure $K_{p} \subset \mathrm{GL}_{2}\left(\mathbb{Z}_{p}\right)$ ) is equivalent to the functor of deformation of a 1-dimensional $p$-divisible group of height 2 over $k$ (resp., with a $\Gamma_{0}(p)$-level structure). Thus, this is exactly the situation arising for elliptic curves and is well understood; c.f. [Buz1]. One concludes for such choice of $K$ that every geometric connected component of the special fibre $\mathbf{M}_{K} \otimes \mathbb{F}_{p}$ of $\mathbf{M}_{K}$ consists of two smooth curves crossing transversely at the supersingular points and so is a normal crossing divisor. Moreover, the natural morphism $\mathbf{M}_{K} \otimes \mathbb{F}_{p} \rightarrow \mathbf{M}_{\mathrm{GL}_{2}\left(\mathbb{Z}_{p}\right) \times K^{p}} \otimes \mathbb{F}_{p}$ is finite flat of degree $p+1$ and admits the usual section taking a false elliptic curve $A$ with $K^{p}$-structure to $\left(A, \operatorname{Ker}\left(\operatorname{Fr}_{A}\right)\right)$ with the same $K^{p}$-structure. The other component is isomorphic to $\mathbf{M}_{\mathrm{GL}_{2}\left(\mathbb{Z}_{p}\right) \times K^{p}} \otimes \mathbb{F}_{p}$ as well. Indeed, the morphism $\mathbf{M}_{K} \otimes \mathbb{F}_{p} \rightarrow \mathbf{M}_{\mathrm{GL}_{2}\left(\mathbb{Z}_{p}\right) \times K^{p}} \otimes \mathbb{F}_{p}$ induces on it a map which is bijective on geometric points (the pre-image of a point $A$ is $\left(A, \operatorname{Ker}\left(\operatorname{Ver}_{A}\right)\right.$ ). Hence the map is purely inseparable of degree $p$.

There is an automorphism $w$ of $\mathbf{M}_{K}$ that is best described by its action on objects: an Iwahori level structure $f: A_{1} \rightarrow A_{2}$ of false elliptic curves is sent by duality to $f^{t}: A_{2}^{t} \rightarrow A_{1}^{t}$. We remark here that every false elliptic curve carries a principal polarization compatible with the $R$-action [Dri2, §4], hence we get $f^{t}: A_{2} \rightarrow A_{1}$, whose isomorphism class is well defined (independent of the choice of polarization). If the kernel of $f$ is connected (resp. étale) then the kernel of $f^{t}$ is étale (resp. connected). It follows that $w$ permutes the two irreducible components of every geometric connected component of $\mathbf{M}_{K} \otimes \mathbb{F}_{p}$. Finally, there is a finite extension $\mathbb{F}_{q} \supseteq \mathbb{F}_{p}$ over which all the connected components of $\mathbf{M}_{K} \otimes \overline{\mathbb{F}}_{p}$ and $\mathbf{M}_{\mathrm{GL}_{2}\left(\mathbb{Z}_{p}\right) \times K^{p}} \otimes \overline{\mathbb{F}}_{p}$ are defined and each connected component is a normal crossing divisor. Using argument as in Remark 3.2, and the fact that $\mathbf{M}_{K} \otimes W\left(\mathbb{F}_{q}\right)$ is flat over $W\left(\mathbb{F}_{q}\right)$ and has reduced special fibre, one find that the connected components of $\mathbf{M}_{K} \otimes \mathbb{F}_{q}\left(\right.$ resp. $\left.\mathbf{M}_{\mathrm{GL}_{2}\left(\mathbb{Z}_{p}\right) \times K^{p}} \otimes \mathbb{F}_{q}\right)$ are in bijection with the connected components of the generic fibre. We conclude that each connected component $Y$ of $\mathbf{M}_{K} \otimes W\left(\mathbb{F}_{q}\right)$ and its image $X \subseteq \mathbf{M}_{\mathrm{GL}_{2}\left(\mathbb{Z}_{p}\right) \times K^{p}} \otimes W\left(\mathbb{F}_{q}\right)$ satisfy the hypotheses of this paper. Moreover, a descent argument, using the uniqueness of the section on each connected component (see Proposition 3.5), allows one to get a section over $\mathbf{M}_{\mathrm{GL}_{2}\left(\mathbb{Z}_{p}\right) \times K^{p}} \otimes \mathbb{Q}_{p}$ defined over $\mathbb{Q}_{p}$. The application of our results gives a new proof for the existence and other properties of canonical subgroups of false elliptic curves, recovering Theorem 11.1 and Lemma 12.5 of [Kas1]. 
5.2. The case $[\boldsymbol{F}: \mathbb{Q}]=\boldsymbol{d}>\mathbf{1}$. In contrast to the previous case, when $F \neq \mathbb{Q}$ there is no natural modular description of the Shimura curves associated to $B$. Instead, by making an auxiliary choice of a CM field $L / F$ in which $\mathfrak{p}$ splits, one can associate to the algebra $B \otimes_{F} L$ another algebraic group $G^{\prime}$ with the same derived group as that of $G$. The curves $M_{K^{\prime}}\left(G^{\prime}, X^{\prime}\right) / F$ associated to $G^{\prime}$ are PEL Shimura curves. These auxiliary curves play an important role in Carayol's construction of an integral model for $M_{K}(G, X) / F$ over $\mathcal{O}=\mathcal{O}_{F, \mathfrak{p}}$, since they are closely related to the Shimura curves defined by $G$ [Car, §4]. Carayol proves that such a model $\mathbf{M}_{K}$ exists, and that there is a universal $p$-divisible $\mathcal{O}$-module $\mathscr{G}$ of $(\mathcal{O}$-) height 2 over the projective limit $\mathbf{M}_{\infty}$ of $\mathbf{M}_{K}$ over $K$. This $p$-divisible group is constructed as a certain "piece" of the $p$-divisible group of the universal abelian variety with additional structure existing over (the projective limit of) the Shimura curves $\mathbf{M}_{K^{\prime}}\left(G^{\prime}, X^{\prime}\right) / \mathcal{O}_{F}$. Note that the $p$-divisible group $\mathscr{G}$ does not carry an $R \otimes \mathcal{O}$-structure. In a moral sense, this structure was already used in reducing the height of the $p$-divisible $\mathcal{O}$-module to 2 (this corresponds to choosing a particular piece of the $p$-divisible group of the universal abelian variety over $\mathbf{M}_{K^{\prime}}\left(G^{\prime}, X^{\prime}\right) / \mathcal{O}_{F}$ and is analogous to the process indicated above for $F=\mathbb{Q}$ ). For details see [Car], in particular $\S \S 3.3,6.3$. We discuss this further.

Assume first that $K=\mathrm{GL}_{2}(\mathcal{O}) \times K^{\mathfrak{p}}$. Thus, no level structure is imposed at $\mathfrak{p}$. Carayol constructs a $p$-divisible group $\mathscr{G}$ over $\mathbf{M}_{\infty}$, which is a $p$-divisible $\mathcal{O}$-module of height 2. For any geometric point $x$ of $\mathbf{M}_{K}$, there is a way to define the fibre $\mathscr{G}_{x}$ by lifting $x$ to a geometric point of $\mathbf{M}_{\infty}$. Over a geometric characteristic 0 point $x$ of $\mathbf{M}_{K}$ we have $\mathscr{G}_{x} \cong\left(F_{\mathfrak{p}} / \mathcal{O}\right)^{2}$. The prime-to-p level structure plays a somewhat dormant role. For example, Carayol proves [Car, §6.6] a "Serre-Tate theorem" to the effect that the formal completion of the henselization of $\mathbf{M}_{K}$ at a geometric point $x$ of its special fibre pro-represents the functor of infinitesimal deformations for the $p$ divisible $\mathcal{O}$-module $\mathscr{G}_{x}$. There are two cases:

(1) The ordinary case, where $\mathscr{G}_{x}$ is isomorphic to $F_{\mathfrak{p}} / \mathcal{O} \oplus\left(F_{\mathfrak{p}} / \mathcal{O}\right)^{t}$, where $(-)^{t}$ denotes the dual $p$-divisible group;

(2) The supersingular case where $\mathscr{G}_{x}$ is the "unique" formal $\mathcal{O}$-module of dimension 1 and height 2 [Dri1, Prop. 1.7].

The deformation theory was worked out by Drinfeld. One concludes that in either case the completed local ring is isomorphic to $\hat{\mathcal{O}}^{\mathrm{nr}} \llbracket t \rrbracket$ and hence that $\mathbf{M}_{K}$ is a regular surface with a smooth special fibre; c.f. [Dri1, Prop. 4.2, 4.5], [Car, App. §3].

Carayol also considers the case of level structure $K_{\mathfrak{p}}(n) \times K^{\mathfrak{p}}$, where $K_{\mathfrak{p}}(n)$ is the subgroup consisting of matrices in $\mathrm{GL}_{2}(\mathcal{O})$ congruent to 1 modulo $\mathfrak{p}^{n}$, and $K^{\mathfrak{p}}$ is small enough. There is a moduli interpretation of a sort to the ensuing morphism $\pi: \mathbf{M}_{K_{\mathfrak{p}}(n) \times K^{\mathfrak{p}}} \rightarrow \mathbf{M}_{K_{\mathfrak{p}}(0) \times K^{\mathfrak{p}}}$; the group scheme $\mathscr{G}\left[\mathfrak{p}^{n}\right]$ descends to $\mathbf{M}_{K_{\mathfrak{p}}(n) \times K^{\mathfrak{p}}}$ and is equipped with a Drinfeld full $\mathfrak{p}^{n}$-level structure, namely, a morphism of $\mathcal{O}$-group schemes $\alpha: \frac{\left(\mathfrak{p}^{-n} / \mathcal{O}\right)^{2}}{\rightarrow} \mathscr{G}\left[\mathfrak{p}^{n}\right]$, such that the closed subscheme $\sum_{P \in\left(\mathfrak{p}^{-n} / \mathcal{O}\right)^{2}} \alpha(P)$ is equal to $\mathscr{G}\left[\mathfrak{p}^{n}\right]$. The scheme $\mathbf{M}_{K_{\mathfrak{p}}(n) \times K^{\mathfrak{p}}}$ is a torsor over 
$\operatorname{Aut}\left(\underline{\left(\mathfrak{p}^{-n} / \mathcal{O}\right)^{2}}\right) \times \mathbf{M}_{K_{\mathfrak{p}}(0) \times K^{\mathfrak{p}}}$ and the morphism $\pi$ is the natural one (in particular its fibres are principal homogenous spaces for $\left.\operatorname{Aut}\left(\left(\mathfrak{p}^{-n} / \mathcal{O}\right)^{2}\right)\right)$. Such level structures were introduced and studied by Drinfeld in [Dri1, p. 572], developed more in [Car, $\S 7$, Appendix], and studied extensively in [KM]. Again Carayol proves a "Serre-Tate theorem" as to the nature of the completed local rings [Car, \$7]. He also proves that the morphism $\pi$ extends the natural morphism $M_{K_{\mathfrak{p}}(n) \times K^{\mathfrak{p}}}(G, X) \rightarrow M_{K_{\mathfrak{p}}(0) \times K^{\mathfrak{p}}}(G, X)$ induced by the inclusion $K_{\mathfrak{p}}(n) \times K^{\mathfrak{p}} \hookrightarrow K_{\mathfrak{p}}(0) \times K^{\mathfrak{p}}$.

As Carayol remarks [Car, §0.4], the construction and results extend to any choice of level subgroup at $\mathfrak{p}$; in particular, for $K=K_{\mathfrak{p}} \times K^{\mathfrak{p}}$, where $K_{\mathfrak{p}}$ is the Iwahori subgroup. The scheme $\mathbf{M}_{K_{\mathfrak{p}} \times K^{\mathfrak{p}}}$ then carries a finite flat group scheme $\mathscr{H}$ (étale locally) with a Drinfeld level structure $\underline{\mathfrak{p}^{-1} / \mathcal{O}} \rightarrow \mathscr{H}$ such that $\sum_{P \in \mathfrak{p}^{-1} / \mathcal{O}} \alpha(P)$ is equal to $\mathscr{H}$ as a closed subscheme. The following conclusion follows from Carayol's work: The completion of the henselization of $\mathbf{M}_{K_{\mathfrak{p}} \times K^{\mathfrak{p}}}$ at a geometric characteristic $p$ point $x$ is the ring that pro-represents the functor of infinitesimal deformations of the divisible $\mathcal{O}$-module $\mathscr{G}_{x}$ together with an $\mathcal{O}$-subgroup scheme of order $q=|\mathcal{O} / \mathfrak{p}|$ killed by $\mathfrak{p}$. This moduli problem can also be phrased in a balanced manner. It can be viewed as deforming a pair of divisible $\mathcal{O}$-modules of height 2 , say $\mathscr{G}_{x}, \mathscr{G}_{x}^{\prime}$, together with an $\mathcal{O}$-isogeny $\mathscr{G}_{x} \rightarrow \mathscr{G}_{x}^{\prime}$ of degree $q$ whose kernel is $\mathfrak{p}$-torsion.

The situation is again very similar to elliptic curves with $\Gamma_{0}(p)$-level structure, and in particular the following holds. The scheme $\mathbf{M}_{K_{\mathfrak{p}} \times K^{\mathfrak{p}}}$ is a regular two dimensional scheme, flat over $\mathcal{O}_{F, \mathfrak{p}}$, the morphism $\pi$ is finite flat of degree $q+1$ and the nature of $\pi$ at every point is completely understood. In particular, there are two pre-images to every ordinary point of $\mathbf{M}_{\mathrm{GL}_{2}(\mathcal{O}) \times K^{\mathfrak{p}}}$ and $\mathbf{M}_{K_{\mathfrak{p}} \times K^{\mathfrak{p}}}$ is regular at each; there is a unique pre-image $y$ to any geometric supersingular point and the completed local ring of $y$ is isomorphic to $\hat{\mathcal{O}}^{\mathrm{nr}} \llbracket s, t \rrbracket /(s t-\varpi)$. For completeness we sketch an argument below. We remark that one can also argue using the results in [Car] obtained for full $\mathfrak{p}$-level structure. However, Carayol uses an explicit description of the formal $\mathcal{O}$-module to obtain his results. Since we do not anticipate such description to be available (or indeed useful) in higher-dimensional cases, using Carayol's result will be contrary to our thesis. We therefore provide an argument that should extend to the more general situation we have in mind.

5.2.1. A sample case. Firstly, we quickly recall the technique of local models in the particular situation of elliptic curves, which serves as a good sample case for our problem.

The deformation theory of elliptic curves (or abelian varieties) can be studied as follows. Given a characteristic $p$ closed point $x$ of a moduli space $\mathbf{M}$ of elliptic curves with level prime to $p$ and its universal object $f: \mathscr{E} \rightarrow \mathbf{M}$, choose an open affine neighborhood $U \ni x$ and a trivialization of $\mathbb{H}_{\mathrm{dR}}^{1}(\mathscr{E} / U) \cong \mathcal{O}_{U}^{2}$. The variation of Hodge structure $R^{0} f_{*} \Omega_{\mathscr{E} / U} \rightarrow \mathbb{H}_{\mathrm{dR}}^{1}(\mathscr{E} / U)$ provides a morphism $U \rightarrow$ Grass, where Grass 
is the Grassmann scheme of locally free, locally direct summands of rank 1 of $\mathcal{O}_{U}^{2}$. One then shows, using the crystalline theory developed by Grothendieck, that this morphism is étale and so is an isomorphism on the level of completed local rings of $x$ and its image in Grass; c.f. [deJ1, DP]. If one wants to work instead with the $p$-divisible groups, one may replace $\mathbb{H}_{\mathrm{dR}}^{1}$ by a similar object provided by the theory of displays as developed by Zink, or by the theory of Cartier-Dieudonné modules, or any other theory studying deformations of $p$-divisible groups. For example, [RZ, §3] choose the Lie algebra of the universal vectorial extension of the $p$-divisible group. By analyzing the Grassmann scheme, one therefore establishes that the completed local ring is $D=W(k(x)) \llbracket t \rrbracket$.

Under this method, the formal scheme representing the infinitesimal deformation problem of an elliptic curve with a subgroup of order $p$ may be translated to a (formal) incidence variety. We think of the moduli problem as the one for a cyclic isogeny $h: E_{1} \rightarrow E_{2}$ of degree $p$ between elliptic curves and we are interested in the completed local ring of the point on the moduli space that corresponds to such data over a finite field $k$ of characteristic $p$. One may choose the trivialization of the two $\mathbb{H}_{\mathrm{dR}}^{1}\left(\mathscr{E} / \operatorname{Spf}\left(D_{i}\right)\right), D_{i}(\cong D)$ the completed local ring at $E_{i}$, such that the isogeny is given by $\left(\begin{array}{ll}1 & 0 \\ 0 & p\end{array}\right)$ [DP, $\S 5.3 \mathrm{ff}$.] or [deJ1]. We are then parameterizing a pair of locally free, locally direct summands $\left(L_{1}, L_{2}\right)$ of rank 1 of $D^{2}$ such that $\left(\begin{array}{ll}1 & 0 \\ 0 & p\end{array}\right) L_{1} \subseteq L_{2}$. In the ordinary case we get an $L_{1}$ whose reduction modulo $p$ is not killed by $\left(\begin{array}{ll}1 & 0 \\ 0 & 0\end{array}\right)$ and the deformation problem is represented by the completion of the local ring of a $k$-point $x$ of $\mathbb{P}_{W\left(\mathbb{F}_{p}\right)}^{1}$ and so is isomorphic to $W(k) \llbracket t \rrbracket$. In the supersingular case we get an $L_{1}$ whose reduction is killed by $\left(\begin{array}{ll}1 & 0 \\ 0 & 0\end{array}\right)$. Let $x$ be a $k$-rational point of $\mathbb{P}_{W(k)}^{1}$ and let $\mathbb{P}$ be the blow-up of $\mathbb{P}_{W(k)}^{1}$ at $x$. Its special fibre has a unique singular point that we shall still denote by $x$. The deformation problem is pro-represented by the completion of the local ring of $x$ on $\mathbb{P}$ and so is isomorphic to $W(k) \llbracket s, t \rrbracket /(s t-p)$.

5.2.2. The calculation of the completed local rings. Recall that the moduli problem is phrased in a balanced manner. Let $x^{\prime}$ be a closed point of $\mathbf{M}_{K_{\mathfrak{p}} \times K^{\mathfrak{p}}}$ with finite residue field $k$, and let $x$ be a $\bar{k}$-point supported on $x^{\prime}$, where $\bar{k}$ is an algebraic closure of $k$. The situation we have is of two divisible $\mathcal{O}$-modules $\mathscr{G}_{x}, \mathscr{G}_{x}^{\prime}$ of dimension 1 and height 2 over $\bar{k}$ and an $\mathcal{O}$-isogeny $h: \mathscr{G}_{x} \rightarrow \mathscr{G}_{x}^{\prime}$ of degree $q=|\mathcal{O} /(\varpi)|$, whose kernel is killed by $\mathfrak{p}$.

Let $\mathscr{G}$ be $\mathscr{G}_{x}$ or $\mathscr{G}_{x}^{\prime}$. The Lie algebra of the universal vectorial extension of $\mathscr{G}$, which serves as a substitute for the first de Rham cohomology, is a free $\bar{k}$-module of dimension 2. As mentioned above, the functor of infinitesimal deformations of $\mathscr{G}$ is pro-representable by $R^{u}=\hat{\mathcal{O}}^{\mathrm{nr}} \llbracket t \rrbracket$, which carries a universal object $\mathscr{G}^{u}$. This can also be proven by the same technique of local models applied to the relative Lie algebra $\underline{\operatorname{Lie}}\left(\mathscr{G}^{u}\right)$ of $\mathscr{G}^{u}$ and the Lie algebra $\underline{\operatorname{Lie}}\left(V \mathscr{G}^{u}\right) \cong\left(R^{u}\right)^{2}$ of its universal vectorial extension $V \mathscr{G}^{u}$, which identifies the completed local ring of $x$ with the completed 
local ring of the $\bar{k}$-point, still called $x$, on the formal Grassmann scheme $\left(\mathbb{P}_{R^{u}}^{1}\right)^{\wedge x}$.

The analogue of Lemma 5.5 of [DP] holds. Namely, one can choose isomorphisms $\underline{\operatorname{Lie}}\left(V \mathscr{G}_{x}^{u}\right) \cong\left(R^{u}\right)^{2}$ and $\underline{\operatorname{Lie}}\left(V \mathscr{G}_{x}^{\prime u}\right) \cong\left(R^{u}\right)^{2}$ such that $h$ is given by the matrix $A=$ $\left(\begin{array}{ll}1 & 0 \\ 0 & \varpi\end{array}\right)$ (one should use that the $p$-divisible groups are in fact polarized, are "special $\mathcal{O}$-modules" in Drinfeld's sense and that $h$ is compatible with the polarizations). Therefore, the completed local ring of $\bar{x}$ is isomorphic to the formal incidence variety in $\left(\mathbb{P}_{R^{u}}^{1}\right)^{\wedge x} \times\left(\mathbb{P}_{R^{u}}^{1}\right)^{\wedge x}$ given by $A$, i.e., by the closed subscheme over which we have $A\left(\underline{\operatorname{Lie}}\left(\mathscr{G}_{x}^{u}\right)\right) \subseteq \underline{\operatorname{Lie}}\left(\mathscr{G}_{x}^{\prime u}\right)$.

In the ordinary case we find that the complete local ring is $\hat{\mathcal{O}}^{\mathrm{nr}} \llbracket t \rrbracket$, and in the supersingular case we find that it is $\hat{\mathcal{O}}^{\mathrm{nr}} \llbracket s, t \rrbracket /(s t-\varpi)$. Finally, one may conclude that the completed local ring of $x^{\prime}$ itself is $\mathcal{O} \otimes_{W(\kappa)} W(k) \llbracket t \rrbracket$ if $x$ is ordinary and is $\mathcal{O} \otimes_{W(\kappa)} W\left(k_{1}\right) \llbracket s, t \rrbracket /(s t-\varpi)$ if $x$ is supersingular, where $\left[k_{1}: k\right] \leq 2$.

Given these results, it is straightforward to verify that the connected components of the generic fibre of a suitable unramified base-change of $\mathbf{M}_{K^{\mathfrak{p}} \times K_{\mathfrak{p}}} \rightarrow \mathbf{M}_{\mathrm{GL}_{2}(\mathcal{O}) \times K^{\mathfrak{p}}}$ satisfy the assumptions of this paper, including the existence of an automorphism $w$. In particular, one has a unique (partial) section on each pair of connected components of the generic fibres; a descent argument allows one to conclude that the section can already be defined before base-change.

Remark 5.1. One may, of course, carry the same analysis for the Shimura curves $M_{K^{\prime}}\left(G^{\prime}, X^{\prime}\right)$. If anything, the analysis is easier, since it is the one underlying Carayol's results. Hence, the results of this paper apply to these cases as well.

Remark 5.2. As is clear from our discussion, whenever we are in a situation of curves $Y \rightarrow X$ such that $Y$ (or the fibres) parameterizes group schemes, e.g. in the case of usual modular curves where $Y$ has a $\Gamma_{0}(p)$-level structure, or for pairs $\mathbf{M}_{K_{\mathfrak{p}} \times K^{\mathfrak{p}}} \rightarrow$ $\mathbf{M}_{K_{\mathfrak{p}}(0) \times K^{\mathfrak{p}}}$ (or the analogous situations for the groups $G^{\prime}$ ), the construction of a section as in this paper provides one with a group scheme over the region where the section is defined. In particular our results reprove Theorems 3.1 and 3.10.7 of [Kat], and Theorem 9.1 of [Kas2] on canonical subgroups of abelian schemes parameterized by $M_{K^{\prime}}\left(G^{\prime}, X^{\prime}\right)$, and in addition provide an analogue of Theorem 3.10.7 of [Kat] for such canonical subgroups.

\section{References}

[AM] A. Abbes, A. Mokrane, Sous-groupes canoniques et cycles évanescents $p$-adiques pour les variétés abéliennes. Inst. Hautes Études Sci. Publ. Math. 99 (2004), 117-162. Zbl 1062.14057 MR 2075884

[AG] F. Andreatta, C. Gasbarri, The canonical subgroup for families of abelian varieties. Preprint, 29 pp., 2003. 
[Ber] P. Berthelot, Cohomologie rigide et cohomologie rigide à support propre. Première partie, Prépublication IRMAR 96-03, 89 pages, 1996.

[BGR] S. Bosch, U. Güntzer, R. Remmert, Non-Archimedean analysis. A systematic approach to rigid analytic geometry. Grundlehren Math. Wiss. 261, Springer-Verlag, Berlin 1984. Zbl 0539.14017 MR 0746961

[BLI] S. Bosch, W. Lütkebohmert, Formal and rigid geometry. I. Rigid spaces. Math. Ann. 295 (1993), 291-317. Zbl 0808.14017 MR 1202394

[BLII] S. Bosch, W. Lütkebohmert, Formal and rigid geometry. II. Flattening techniques. Math. Ann. 296 (1993), 403-429. Zbl 0808.14018 MR 1225983

[Buz1] K. Buzzard, Integral models of certain Shimura curves. Duke Math. J. 87 (1997), 591-612. Zbl 0880.11048 MR 1446619

[Buz2] K. Buzzard, Analytic continuation of overconvergent eigenforms. J. Amer. Math. Soc. 16 (2003), 29-55. Zbl 01832407 MR 1937198

[Car] H. Carayol, Sur la mauvaise réduction des courbes de Shimura. Compositio Math. 59 (1986), 151-230. Zbl 0607.14021 MR 0860139

[Co] R. Coleman, On the components of $X_{0}\left(p^{n}\right)$. J. Number Theory 110 (2005) 3-21. Zbl 02142233 MR 2114670

[CGJ] R. Coleman, F. Gouvêa, N. Jochnowitz, $E_{2}, \Theta$, and overconvergence. Internat. Math. Res. Notices 1995 (1995), 23-41 (electronic). Zbl 0846.11027 MR 1317641

[Con] B. Conrad, Higher-level canonical subgroups in abelian varieties. Preprint, 2005.

[DP] P. Deligne, G. Pappas, Singularités des espaces de modules de Hilbert, en les caractéristiques divisant le discriminant. Compositio Math. 90 (1994), 59-79. Zbl 0826.14027 MR 1266495

[DT] F. Diamond, R. Taylor, Nonoptimal levels of mod $l$ modular representations. Invent. Math. 115 (1994), 435-462. Zbl 0847.11025 MR 1262939

[Dri1] V. G. Drinfeld, Elliptic modules. Mat. Sb. (N.S.) 94 (136) (1974), 594-627, 656. Zbl 0321.14014 MR 0384707

[Dri2] V. G. Drinfeld, Coverings of p-adic symmetric domains. Funkcional. Anal. i Priložen. 10 (2) (1976), 29-40. Zbl 0346.14010 MR 0422290

[deJ1] A. J. de Jong, The moduli spaces of principally polarized abelian varieties with $\Gamma_{0}(p)$ level structure. J. Algebraic Geom. 2 (1993), 667-688. Zbl 0816.14020 MR 1227472

[deJ2] A. J. de Jong, Crystalline Dieudonné module theory via formal and rigid geometry. Inst. Hautes Études Sci. Publ. Math. 82 (1995), 5-96; Erratum: ibid. 87 (1998), 175. Zbl 0864.14009 MR 1383213; MR 1659266

[Kas1] P. L. Kassaei, p-adic modular forms over Shimura curves over $\mathbb{Q}$. Ph.D. Thesis, MIT, 1999.

[Kas2] P. L. Kassaei, $\mathscr{P}$-adic modular forms over Shimura curves over totally real fields. Compositio Math. 140 (2004), 359-395. Zbl 1052.11037 MR 2027194

[Kas3] P. L. Kassaei, A gluing lemma and overconvergent modular forms. To appear in Duke Math J. 132. 
[Kat] N. M. Katz, p-adic properties of modular schemes and modular forms. In Modular functions of one variable, III (Proc. Internat. Summer School, Univ. Antwerp, Antwerp, 1972), Lecture Notes in Math. 350, Springer-Verlag, Berlin 1973, 69-190. Zbl 0271.10033 MR 0447119

[KM] N. M. Katz, B. Mazur, Arithmetic moduli of elliptic curves. Ann. of Math. Stud. 108. Princeton University Press, Princeton, NJ, 1985. Zbl 0576.14026 MR 0772569

[KL] M. Kisin, K. F. Lai, Overconvergent Hilbert Modular forms. Amer. J. Math. 127 (2005), 735-783. Zbl 02208014 MR 2154369

[Nev] E. Nevens, The Hecke operator $U_{p}$ for overconvergent Hilbert modular forms. Thesis. Imperial College, London 2003.

[RZ] M. Rapoport, Th. Zink, Period spaces for p-divisible groups. Ann. of Math. Stud. 141, Princeton University Press, Princeton, NJ, 1996. Zbl 0873.14039 MR 1393439

[SGA4] Théorie des topos et cohomologie étale des schémas. Séminaire de Géométrie Algébrique du Bois-Marie 1963-1964 (SGA 4). Dirigé par M. Artin, A. Grothendieck et J. L. Verdier. Avec la collaboration de N. Bourbaki, P. Deligne et B. Saint-Donat. Lecture Notes in Math. 270, Springer-Verlag, Berlin 1972. Zbl 0237.00012 MR 0354653

[Tat] J. Tate, Rigid analytic spaces. Invent. Math. 12 (1971), 257-289. Zbl 0212.25601 MR 0306196

Received January 7, 2005

Eyal Z. Goren, Department of Mathematics and Statistics, McGill University, 805 Sherbrooke West, Montreal H3A 2K6, Quebec, Canada

E-mail: goren@math.mcgill.ca

Payman L. Kassaei, Department of Mathematics and Statistics, McGill University, 805 Sherbrooke West, Montreal H3A 2K6, Quebec, Canada

E-mail: kassaei@math.mcgill.ca 